\title{
The Dynamics of Argumentative Discourse
}

\author{
Alexander W. Kocurek ${ }^{1} \cdot$ Carlotta Pavese $^{1}$ (D)
}

Received: 26 March 2021 / Accepted: 7 September 2021 /Published online: 25 October 2021

(C) The Author(s), under exclusive licence to Springer Nature B.V. 2021

\begin{abstract}
Arguments have always played a central role within logic and philosophy. But little attention has been paid to arguments as a distinctive kind of discourse, with its own semantics and pragmatics. The goal of this essay is to study the mechanisms by means of which we make arguments in discourse, starting from the semantics of argument connectives such as 'therefore'. While some proposals have been made in the literature, they fail to account for the distinctive anaphoric behavior of 'therefore', as well as for uses of argument connectives in complex arguments, suppositional arguments, arguments with non-declarative conclusions, as well as arguments with parenthetical remarks. We argue that a comprehensive account of arguments requires imposing a distinctive tree-like structure on contexts. We show how to extend our account to accommodate modal subordination and different flavors of argument connectives.
\end{abstract}

Keywords Arguments - Anaphora - Dynamic Semantics · Therefore - Supposition · Modal subordination

\section{Introduction}

In natural languages, arguments are conventionally associated with particular grammatical constructions, such as:

(1) a. $P_{1}, \ldots, P_{n}$. Therefore, $C$;

b. Suppose $P_{1}, \ldots, P_{n}$. Then, $C$.

These constructions involve words such as 'therefore', 'then', 'thus', 'hence', 'so', etc. - or ARGUMENT CONNECTIVES. While some proposals have been made about

The order of the authors is alphabetical. The authors contributed equally to this work.

Carlotta Pavese

cp645@cornell.edu

Alexander W. Kocurek

awk78@cornell.edu

1 Sage School of Philosophy, Cornell University, Goldwin Smith Hall, Ithaca, 14850, NY, USA 
the semantics of 'therefore' and related expressions in the recent literature $[15,59$, 60], these proposal focus narrowly on simple arguments with categorical premises and declarative conclusions. These proposals fail to account for uses of argument connectives in arguments with non-declarative conclusions, in suppositional arguments, and in complex arguments - arguments that contain subarguments. Our primary goal in this paper is to provide a comprehensive semantics for argument connectives that captures their uses in argumentative discourses.

Because arguments are made through discourses, it is natural to appeal to dynamic approaches to meaning in order to model arguments in discourse. Our analysis will be deeply informed by such approaches - specifically by dynamic approaches to modals and conditionals (e.g., [10, 11, 15, 26, 35, 53, 74, 75, 84, 85, 93]). As we will see, however, in order to capture the distinctive dynamics of arguments, we need to enrich our model of contexts beyond what is typically assumed in dynamic semantics - viz., as bodies of information (e.g., as a set of worlds), perhaps imbued with some additional structure (e.g., a probability function, discourse referents, a preference ordering, a partition, etc.). The reason is that arguments with multiple suppositions (as in proof by cases) or suppositions within suppositions (as in conditional proofs) seem to make use of multiple bodies of information at once. So contexts need to keep track of several bodies of information, as well as of how these bodies of information relate to one another within an argument. One upshot of this project is that the notion of context required to model the dynamics of arguments has a distinctive tree-like structure - a structure similar to what has been proposed in SDRT, which however has been developed to account primarily for pronominal anaphora [7, 36, 46].

Here is the plan going forward. In Section 2, we present some initial data surrounding argument connectives like 'therefore' that we wish to capture. Having outlined the data, our approach will be incremental. In Section 3, we introduce a simple dynamic semantics that can model categorical arguments - i.e., arguments that have categorical, rather than suppositional, premises - and we show how this simple semantics can be augmented to account for arguments with non-declarative conclusions. In Section 4, we extend this framework to account for suppositional arguments as well as complex arguments - i.e., arguments that contain subarguments. In Section 5, we refine this semantics further so to capture modal subordination effects in suppositional arguments as well as different flavors of 'therefore'. We conclude in Section 6 with some directions for future research.

\section{The Data}

\subsection{Anaphoric Behavior}

A central observation to our project is that argument connectives such as 'therefore' exhibit an anaphoric behavior [15, p. 296]; [92]. ${ }^{1}$ An anaphoric expression is one

\footnotetext{
${ }^{1}$ Argument connectives have an anaphoric component not only in English ('there' in 'therefore') but also in German and Dutch - i.e., 'des' in 'deshalb' and 'daar' in 'daarom'. Thanks to Mats Rooth for discussion.
} 
whose referent is supplied by an occurrence of some other antecedent expression [39]. The standard diagnostics are (i) anaphors need a linguistic antecedent; (ii) they can be interpreted relative to different antecedents depending on the context; and (iii) anaphors can occur in donkey-like sentences.

'Therefore' satisfies these main criteria for anaphoricity. First, it needs a linguistic antecedent, as evinced by the infelicity of the following (said out of the blue): ${ }^{2}$

(2) a. ??Therefore/Hence/Thus, we should leave.

b. ??Therefore/Hence/Thus, streets are wet.

c. ??Therefore/Hence/Thus, either it is raining or it is not raining.

Indeed, while it is a familiar observation that epistemic modals such as 'must' can also be anaphoric (on arguments, cf. [81]), the anaphoric behavior of 'therefore' is even more explicit than that of 'must', in that while 'therefore' requires a linguistic antecedent, 'must' does not:

(3) a. [Looking at the clock.] We must leave!

b. [Looking out the window at the rain.] The streets must be wet.

c. [The logic instructor says:] It must be that either it is raining or it is not raining.

The antecedent of 'therefore' can be a premise, or a list of premises:

(4) a. There is an on-going epidemic crisis. Therefore, we need vaccines.

b. It is raining. Hence, the streets are wet.

c. I am smelling gas in the kitchen. Thus, there is a gas leak.

It can also be a whole argument, as in the following examples:

(5) Suppose it is raining. Then the streets are wet. Therefore, if it is raining, the streets are wet.

(6) Maria is either from Turin or from Madrid. Suppose she is from Turin. Then she is Italian. Suppose instead she is from Madrid. Then she is Spanish. Therefore, she is either Italian or Spanish.

(7) Suppose there is a largest prime number $p$. Then $p$ ! +1 is larger than $p$. But $p !+1$ is prime, contradiction. Therefore, there is no largest prime number.

Like other anaphors, the linguistic antecedent for argument connectives does not need to be the most immediate one. For example, in (8a), it is natural to take the antecedent

\footnotetext{
${ }^{2}$ [55, pp. 399-406] argues that 'therefore' is deictic. However, the need for a linguistic antecedent suggests instead that argument connectives are better modeled as anaphors. The use of 'so' strikingly differs from the use of 'therefore' in this regard, in that 'so' can also be used without premises, as in "So, you have arrived!". This strikes us as meaning something quite different from 'therefore', however.
} 
of 'therefore' to be the categorical premise that Mark went to the grocery store and in (8b), the natural antecedent of 'then' is the suppositional premise 'Suppose Mark went to the grocery store this morning':

(8) a. Mark went to the grocery store this morning. (Have you been? They have all sorts of exotic fruit.) Therefore, he has not stayed all day at home.

b. Suppose Mark went to the grocery store this morning. (Have you been? They have all sorts of exotic fruit.) Then he bought dragon fruit.

Like anaphors, it can be ambiguous what the antecedent is. For example, in (9), there are two possible antecedents for the last sentence.

(9) Either it is raining or it is not. Suppose it is raining. Then you should take the umbrella. Suppose it is not raining. Then taking the umbrella will do no harm. Therefore, you should take an umbrella.

a. CATEGORICAL: you should take an umbrella regardless of whether it is raining or not.

b. SUPPOSITIONAL: you should take an umbrella also assuming it is not raining.

On the most natural reading — which we will call the CATEGORICAL reading — the antecedent is the entire argument from the premise 'Either it is raining or it is not' to 'Then taking the umbrella will do no harm'. However, there is another reading what we will call the SUPPOSITIONAL reading - where the antecedent of the last 'therefore' is the subargument 'Suppose it is not raining. Then taking the umbrella will do no harm'. On this reading, the last sentence only expresses a claim about what follows from the supposition that it is not raining. (This reading can be brought out by continuing (9) with '... So either way, you should take an umbrella.')

Finally, the following are plausible examples of donkey sentences involving 'therefore':

(10) a. Whenever one believes a certain view, one has to believe that its consequences are therefore true.

b. If one derives a contradiction from a claim, one may infer that it is therefore false.

Taken together, these observations suggest that 'therefore' exhibits similar behavior to anaphors.

This said, the anaphoric behavior of 'therefore' is, in some respects, more constrained than ordinary pronominal anaphora. Pronouns can anaphorically depend on very far back antecedents, if they are sufficiently salient; by contrast, like propositional anaphora more generally [43], it seems to be more constrained in how far back 'therefore' can reach its antecedents and so in the sort of antecedents it can take. 
For example, in (11), 'therefore' cannot take 'The wall is wet with paint' only as an antecedent. $^{3}$

(11) The wall is wet with paint and it is made of concrete. ??Therefore, you will stain your shirt if you lean on it.

Moreover, piled up 'therefore' can only refer to the immediately precedent ones. For example, in (12), the last 'therefore' cannot select only 'Paolo is Italian' as its antecedent and hence is off when its conclusion is not relevant to its immediate precedent.

(12) Paolo is Italian. Therefore, he is European. ?Therefore, he speaks Italian.

While there is more work to be done to sort out what exactly the restrictions on the anaphoric behavior of 'therefore' are, the important observation for our purposes is that it has anaphoric behavior in the first place. ${ }^{4}$

\subsection{Embedding and Projection}

A second observation about 'therefore' is that it appears to have a restrictive syntax in its embedding behavior. On one hand, 'therefore' can embed under conjunction as in (13).

(13) It is raining and therefore the streets are wet.

But, for example, it cannot directly embed under disjunction:

(14) There are dark clouds on the horizon. ??Either it is therefore raining or it is therefore snowing.

Moreover, 'therefore' seems to embed under modals but when it does, it privileges a wide scope reading. Thus, in the examples in (15), the modals are naturally interpreted as having narrow scope with respect to 'therefore':

(15) It's raining...

a. The streets might therefore be wet.

$\approx$ Therefore, the streets might be wet.

b. The streets are probably, therefore, wet.

$\approx$ Therefore, the streets are probably wet.

c. It must be that therefore the streets are wet.

$\approx$ Therefore, the streets must be wet.

The same is true for negation: it seems that even when negation syntactically scopes over 'therefore', it is naturally interpreted as having narrow scope under 'therefore'

\footnotetext{
${ }^{3}$ Thanks to Mats Rooth for bringing this important point to our attention.

${ }^{4}$ In being more constrained than pronominal anaphora, the anaphoric behavior of 'therefore' resembles null anaphora ([18, p. 191]; [19, p. 21]; [50, pp. 246-8]). More generally, propositional anaphora seems to be more constrained than pronominal anaphora in the sort of antecedents that it can take (e.g., [24, 43, 54]).
} 
(as illustrated in (16a)) and that 'therefore' cannot easily be embedded under negation (as illustrated in (16b)).

(16) a. It is not, therefore, raining. $\approx$ Therefore, it is not raining.

b. ??It is humid outside. It is not the case that therefore the street are wet.

With that said, we can force modals and negation to take wide scope over 'therefore' by embedding the 'therefore' in a conjunction.

(17) a. It might be that it is raining and therefore the streets are wet.

b. It is probable that it is raining and therefore the streets are wet.

c. It must be that it is raining and therefore the streets are wet.

d. It is not the case that Mark is a progressive and therefore a supporter of the Green New Deal.

Yet, although 'therefore' can, syntactically, take narrow scope with respect to modals and negation when it immediately embeds under conjunction, semantically the relation between the antecedent and the consequent can project out of the embeddings $[55,59,60,80]$. Thus, $(17 \mathrm{~d})$ has a reading which conveys that Mark's supporting the Green New Deal follows from his being a progressive. ${ }^{5}$ Similarly for these examples:

(18) a. Is Mark a progressive and therefore a supporter of the Green New Deal?

b. If Mark is a progressive and therefore a supporter of the Green New Deal, he will not vote for Trump.

c. It might be that Mark is a progressive and therefore a supporter of the Green New Deal.

Moreover, as famously observed by [29], the entailment expressed by 'therefore' cannot easily targeted by demonstratives or denied, suggesting that it is not at issue even when used outside of conjunctions. Indeed, in this respect 'therefore' strikingly differs from 'it follows that':
a. A: Mary is English and therefore brave.
$\mathrm{B}$ : \#That is false. [where 'that' targets the entailment]
b. A: Mary is English and it follows from this that she is brave. B: That is false.

\footnotetext{
${ }^{5}$ We think that non-projective readings under modals, belief reports, and negation are also sometimes possible (for some discussion of non-projective readings under negation, see [60]), and that our proposed analysis can be extended to capture those too. However, because these embedded uses of 'therefore' are not our main focus in this paper, we bracket this big issue here. We will return to non-projective readings under modals and belief reports briefly in Section 3.2.
} 
Furthermore, in contrast with what we have observed with 'therefore', the entailment cannot project out of embeddings when it is conveyed by 'it follows that':

(20) a. It is not the case that Mary is English and that it follows from that that she is brave.

b. Is it the case that Mary English and that it follows from this that she is brave?

While Grice has argued on these bases that 'therefore' is a paradigmatic example of a conventional implicature, some have argued that the pattern of projection is actually more constrained than that associated typically with conventional implicatures. While appositives, for example, are widely projective even when embedded in attitude reports (e.g., 'Mary believes that June, who is English, is brave' conveys that June is English; cf. [51, 65]), with attitude verbs, and perhaps with epistemic modals too, both the projective and the non-projective reading of 'therefore' seem to be available (e.g., 'Mary believes that June is English and therefore brave'). Even within the scope of negation, some hear both a projective and a non-projective reading for 'therefore'. On the basis of this and other evidence, 'therefore' seems to behave more like a presupposition trigger (cf. [60]). ${ }^{6}$

\subsection{Non-Declarative Premises/Conclusions}

A third observation is that not only declarative sentences but also imperatives and interrogatives can appear as conclusions of arguments (cf. [17, 57, 58, 76]):

(21) a. If May arrives late tonight, you should go to the store. As a matter of fact, Mary is arriving late. Therefore, go to the store!

b. But seek first the kingdom of God and His righteousness, and all these things will be added unto you. Therefore do not worry about tomorrow, for tomorrow will worry about itself. Today has enough trouble of its own. (Matthew 3:64)

(22) a. The victim was stabbed to death. Therefore, who committed the murder?

b. Whoever is faithful with very little will also be faithful with much, and whoever is dishonest with very little will also be dishonest with much. Therefore, if you have not been faithful with worldly wealth, who will entrust you with true riches? (Luke 16:11)

\footnotetext{
${ }^{6}$ The boundaries between conventional implicatures and presuppositions are notoriously hard to draw and some have doubts about there being a fruitful distinction here $[8,33]$. In this paper, we are more concerned with the task of modeling the phenomenon than with the task of classifying it.
} 
By contrast, the data is less clear about whether the antecedents of an argument connective can be imperative or interrogative. For example, the following discourses sound off:

(23) a. Go to the store. ??Therefore, take the car.

b. Go to the store. ??Therefore, you can't go see your friends.

(24) a. Have you seen my keys? ??Therefore, where did I put them?

b. Have you seen my keys? ??Therefore, I've lost them.

In the light of this data, in our discussion, we will proceed on the assumption that, while the conclusions of an argument can be non-declarative, the premises of an argument ought to be declarative.

\section{A Semantics for Categorical Arguments}

Having examined some data surrounding argument connectives that it is important for a theory of arguments to capture, we will start from a simple semantics for categorical arguments inspired by [59] dynamic semantics for 'therefore'. We begin in Section 3.1 with a brief motivation for this account of 'therefore' by noting how it compares with epistemic 'must'. Then in Section 3.2, we present the dynamic account more formally. In Section 3.3, we discuss different notions of entailment one could define in this framework. Finally, in Section 3.4, we show how to extend this account to cover categorical arguments with non-declarative conclusions.

\subsection{Analogies and Contrasts Between 'therefore' and Epistemic Modals}

As observed by [15, pp. 295-6], 'therefore' bears a close resemblance with modals. First, (25) is very close in meaning to the modalized conditional (26):

(25) Sarah saw a puppy. Therefore, she petted it.

(26) If Sarah saw a puppy, she (obviously/necessarily/must have) petted it.

provided that we add to (26) the premise (27):

(27) Sarah saw a puppy.

Second, as we have seen in Section 2.1, 'therefore' exhibits an anaphoric behavior, similar to that of modals. Third, just like modals can come in different flavors (causal, logical consequence, practical) [41, 42], so can 'therefore'. In particular, as discourse coherence theorists have emphasized, 'therefore' can be used with a causal meaning (cf. [6, 7, 12-14, 32, 36]), as in (28).

(28) a. Mary qualified for the exam. Therefore, she could enroll.

b. A lighted cigarette was carelessly dropped into the hay. Therefore, the hay was caught on fire. 
And arguments can also have practical flavor (cf. [15, p. 279]), as in (29).

(29) We cannot put the face of a person on a stamp unless said person is deceased. My suggestion, therefore, is that you drop dead (attributed to J. Edward Day; letter, never mailed, to a petitioner who wanted himself portrayed on a postage stamp).

While this analogy between 'therefore' and modals is important and instructive, one important difference is worth highlighting. As we have seen in Section 2.2, 'therefore' exhibits a projective behavior. By contrast, 'must' does not have the same projective behavior. None of $(30 \mathrm{a}-\mathrm{d})$ conveys that Mark's supporting the Green New Deal follows in any way from him being a progressive:

(30) a. It is not the case that Mark is a progressive and must support the Green New Deal.

b. Is Mark a progressive and must support the Green New Deal?

c. If Mark is a progressive and must support the Green New Deal, he will not vote for Trump.

d. It might be that Mark is a progressive and must support the Green New Deal.

So, while a semantics for 'therefore' should aim at capturing the analogy with modals observed by [15], it also should account for this difference in projective behavior.

\subsection{Dynamic Semantics for 'therefore'}

\subsubsection{Syntax}

We introduce a simple language containing the standard Booleans, epistemic 'must' and 'might', and the argument connective $\therefore$ We will formalize all conclusion connectives — 'therefore', 'thus', 'then', etc. — using $\therefore$. While there are some notable differences between these connectives (see Section 6), we set them aside here.

Definition 1 (Simple Syntax)

$$
\begin{aligned}
& \phi::=p|\neg \phi|(\phi \wedge \phi)|(\phi \wedge \therefore \phi)|(\phi \vee \phi)|(\phi \rightarrow \phi)| \diamond \phi \mid \square \phi \\
& \sigma::=\phi \mid \therefore \phi
\end{aligned}
$$

This syntax only allows $\therefore$ to occur within certain embedded contexts, specifically within conjunction, or within other operators embedding conjunctions:

$$
\begin{aligned}
& p \wedge \therefore q \\
& \neg(p \wedge \therefore q), \text { etc. }
\end{aligned}
$$

This restrictive syntax reflects the fact that, as we have seen in Section 2.2, 'therefore' cannot directly embed under any operator - e.g., it cannot directly embed under disjunction, as in (14); and it is naturally interpreted as having wide scope over 
epistemic modals and negation, as narrow-scope readings, when possible, are rather forced (cf. $(15 a-c) ;(16 a-b))$.

It would of course be desirable to have some explanation for this restrictive syntax rather than just to stipulate it. One possible explanation (for at least some of this restricted syntactic distribution) is that drawing a conclusion is a performative speech act. If 'therefore' encodes a performative element, we should expect it to behave similarly to performatives such as 'I hereby conclude'. And performatives such as 'I hereby conclude' are indeed similar to 'therefore' in their restricted syntactic distribution. ${ }^{7}$ For one thing, they typically do not embed under disjunction but, like 'therefore', they can embed under conjunction [44]:

(31) a. There are dark clouds on the horizon. ?I hereby conclude that it is raining, or I hereby conclude that it is not raining.

b. There are dark clouds on the horizon and I hereby conclude that it is raining.

Similar considerations might explain the observation that 'therefore' is naturally interpreted as taking wide scope over epistemic modals and negation. After all, sentences in which 'I hereby conclude' is embedded under epistemic modals or negation are also rather forced: ${ }^{8}$

(32) a. It is raining. ?It might be that I hereby conclude that the streets are wet.

b. It is raining. ?It is not the case that I hereby conclude that the streets are wet.

The assumption that argument connectives such as 'therefore' encode a performative element might explain their restricted syntax and so motivates our syntax as well as the focus of our analysis going forward. ${ }^{9}$

\subsubsection{Semantics}

We propose a dynamic analysis on which 'therefore' works as a particular kind of test. In dynamic semantics, a test is an expression whose role is to check that the context satisfies certain constraints as opposed to adding information to the context.

\footnotetext{
${ }^{7}$ We thank a referee for this helpful suggestion.

${ }^{8}$ One potential difference in this respect is that performatives such as 'I hereby promise' and 'I hereby conclude' can be denegated [31, 71] as in (i):

(i) a. I don't conclude that he is wrong.

b. I don't promise that I will behave.

By contrast, it is not clear that there is an analogous denegative use for 'therefore' under negation.

${ }^{9}$ Another possible explanation of the fact that 'therefore' does not easily embed under negation might be syntactic: as is well-known, negation creates scope islands ([68]), and so the antecedent of 'therefore' ought to occur within the island for 'therefore' to be anaphorically dependent on it. This might explain why $\neg(\phi \wedge \therefore \psi)$ is grammatical whereas $\neg(\therefore \phi \wedge \psi)$ is not. Thanks go to a helpful referee for this suggestion.
} 
The simplest examples of dynamic entries for 'might' [85], 'must' [89], and the conditional $[27,74,84]$ are as follows:

$$
\begin{aligned}
s[\nabla \phi] & =\{w \in s \mid s[\phi] \neq \varnothing\} \\
s[\square \phi] & =\{w \in s \mid s[\phi]=s\} \\
s[\phi \rightarrow \psi] & =\{w \in s \mid s[\phi][\psi]=s[\phi]\} .
\end{aligned}
$$

Here, $s$ is an INFORMATION STATE - i.e., a set of possible worlds. According to the entries above, 'might', 'must', and 'if... then' test whether $s$ satisfies certain properties. In the case of 'might', it tests whether $s$ is consistent with the prejacent $\phi$. In the case of 'must', it tests whether $s$ "supports" $\phi-$ i.e., whether $s[\phi]=s$. In the case of 'if $\phi$, then $\psi$ ', it tests whether the result of updating $s$ with $\phi$ supports $\psi$. In each case, if $s$ passes the test, it leaves $s$ unchanged; otherwise, it returns the empty set.

The general idea is that 'therefore' tests that the current state already supports the conclusion, similarly to 'must'. The only difference is that when a state does not pass this test, the result is not the empty set but an undefined value.

$$
s[\therefore \phi]= \begin{cases}s & \text { if } s[\phi]=s \\ \text { undefined otherwise }\end{cases}
$$

This clause models 'therefore' in the way presupposition triggers are typically modeled in dynamic semantics (cf. [10]). ${ }^{10}$

This captures the difference in projective behavior between 'therefore' and 'must'. Consider again $(17 \mathrm{~d})$ :

(17d) It is not the case that Mark is a progressive and therefore a supporter of the Green New Deal.

This sentence's update effect is only defined when updating with Mark being a progressive results in a state that supports Mark's supporting the Green New Deal. On a standard dynamic treatment for negation, it takes the complement of the result of updating with the prejacent:

$$
s[\neg \phi]=s-s[\phi] .
$$

Hence, if 'therefore' returned the empty set as the result of a failed test, then the result of negating it would be defined as well:

$$
s[\neg(\psi \wedge \therefore \phi)]=s-s[\psi][\therefore \phi]=s-\emptyset=s .
$$

By contrast, if 'therefore' returns an undefined value as the result of a failed test, then the result of negating it is also undefined: the complement of an undefined value is undefined. And if we want to allow the content to project, the value of a failed test must be undefined.

Thus, we can give a full statement of the update effects of sentences on information states as follows.

\footnotetext{
${ }^{10}$ Indeed, this entry for $\therefore$ is exactly like the entry [10, pp. 156-162] assigns to the presuppositional operator $\delta$. Like our $\therefore, \delta$ returns the context if it supports its prejacent, else it returns an undefined value. The differences between $\delta$ and $\therefore$ will become clear in the next section, where we tackle how to model the anaphoric behavior of 'therefore'.
} 
Definition 2 (Simple Dynamic Semantics) Where $s \subseteq W$ is an information state:

$$
\begin{aligned}
s[p] & =\{w \in s \mid w(p)=1\} \\
s[\neg \phi] & =s-s[\phi] \\
s[\phi \wedge \psi] & =s[\phi][\psi] \\
s[\phi \vee \psi] & =s[\phi] \cup s[\psi] \\
s[\square \phi] & =\{w \in s \mid s[\phi]=s\} \\
s[\diamond \phi] & =\{w \in s \mid s[\phi] \neq \varnothing\} \\
s[\phi \rightarrow \psi] & =\{w \in s \mid s[\phi][\psi]=s[\phi]\} \\
s[\therefore \phi] & = \begin{cases}s & \text { if } s[\phi]=s \\
\text { undefined otherwise }\end{cases}
\end{aligned}
$$

On this semantics, 'therefore' is a test. ${ }^{11}$ As such, it does not add information to the context. This seems the desired analysis to us: taking 'therefore' to be informative would not quite capture the functional role that we think is associated with 'therefore'. Conceptually, we take the function of 'therefore' to be to highlight information that is already available, as it happens in proofs. If 'therefore' were to add information to the context, its role of highlighting consequences of the current context would be lost. While treating 'therefore' as a sui generis test accounts for this intuition, treating 'therefore' as an informative update does not. Furthermore, we want to distinguish adding premises from drawing a conclusion. If we were to think of 'therefore' as informative, we would miss out on the difference between categorical assertions and conclusions.

This is not to say that 'therefore' can never be informative. Although this theory of 'therefore', like dynamic theories of 'might' and 'must', does not treat sentences of the form 'Therefore $\phi$ ' as informative, one can adopt solutions that have been made available elsewhere to capture informative uses of 'therefore'. For example, [93] accounts for informative uses of 'might' by modeling information states not as sets of worlds but as sets of sets of worlds; updating an information state with $\diamond \phi$ then amounts to removing all sets of worlds that do not leave open $\phi$. Similarly, one could model informative uses of $\therefore \phi$ by treating information states as sets of sets of worlds and then stipulate that $\therefore \phi$ removes all sets of worlds that do not support $\phi$. Another option is to explain informative uses of 'therefore' in terms of repair mechanisms, such as presupposition accommodation (e.g., [9, 49, 72, 88]). So, while

\footnotetext{
${ }^{11}$ One potential objection to this semantics for 'therefore' is that because it treats 'therefore' as a test with the same strength as [89] 'must', it predicts that adding 'must' in a conclusion with 'therefore' should be redundant. But intuitively, that is not the case; e.g., in (i), 'must' is nonredundant:
}

(i) Mark owns a Bentley. Therefore, he must be rich.

Following [47] and [23] (and against [89] and [90]), we think it is far from obvious that 'must' is as strong as 'therefore'. Regardless of this thorny issue, the same objection would apply to test semantics for conditionals $([26,74,84])$, as e.g., the 'must' in "If Mark owns a Bentley, then he must be rich" also seems nonredundant. For a plausible account of what (a strong) 'must' adds that is compatible with our approach, see [52]. 
we do not take informative uses of 'therefore' to be paradigmatic, there are several resources at our disposal to model them.

Example 3 (Embedding Under Negation) Consider (17d) again. This sentence has the form $\neg(p \wedge \therefore$ gnd $)$. Intuitively, (17d) conveys that Mark's supporting the Green New Deal follows from his being progressive. Indeed, this is exactly what our semantics predicts. The update effect of $\neg(p \wedge \therefore$ gnd $)$ on a state $s$ is as follows:

$$
\begin{aligned}
s[\neg(p \wedge \therefore g n d)] & =s-s[p \wedge \therefore g n d] \\
& =s-s[p][\therefore g n d] \\
& = \begin{cases}s-s[p] & \text { if } s[p][g n d]=s[p] \\
\text { undefined } & \text { otherwise }\end{cases}
\end{aligned}
$$

This shows that the entailment projects out of negation in such a way that the sentence is felicitous only if Mark's supporting the Green New Deal follows from his being progressive. $^{12}$

\subsection{Entailment}

In dynamic semantics, there are generally at least two notions of entailment one can define: static and dynamic. The choice between them amounts to whether we only require the conclusion to be supported on states that already support the premises (static) or on any state updated with the premises (dynamic). We can state these notions more formally as follows: ${ }^{13}$

Definition 4 (Static Entailment, $\vDash_{\mathrm{s}}$ ) $\phi_{1}, \ldots, \phi_{n} \vDash_{\mathrm{s}} \psi$ iff for all information states $s$, if $s\left[\phi_{i}\right]=s$ for each $1 \leq i \leq n$, then $s[\psi]=s$.

Definition 5 (Dynamic Entailment, $\vDash_{\mathrm{d}}$ ) $\phi_{1}, \ldots, \phi_{n} \vDash_{\mathrm{d}} \psi$ iff for all information states $s$, if $s\left[\phi_{1}\right] \cdots\left[\phi_{n}\right]$ is defined, then $s\left[\phi_{1}\right] \cdots\left[\phi_{n}\right][\psi]=s\left[\phi_{1}\right] \cdots\left[\phi_{n}\right]$.

The difference comes out clearly when looking at epistemic modals. For example, $\diamond \phi, \neg \phi \vDash_{\mathrm{s}} \perp$, since if $s[\diamond \phi]=s$, then $s[\neg \phi] \neq s$. By contrast, $\diamond \phi, \neg \phi \nvdash_{\mathrm{d}} \perp$. For

\footnotetext{
${ }^{12}$ Similarly, as we have seen, $\therefore$ can project out of epistemic modals and our test semantics can easily be extended to capture those projective readings. Some speakers report the availability of a non-projective reading of 'therefore' under 'might'. We could complicate the current system to allow for 'therefore' to embed under epistemic modals and by introducing an accessibility for ' $\diamond$ ' (cf. [28]). Then we could model the different readings as testing different accessibility sets. Indeed, this general strategy would also enable us to capture non-projective readings of 'therefore' under belief reports (Section 2.2, footnote 5). However, here we set this complexity aside and for simplicity, we will stick with our simple system without accessibility relations in what follows.

${ }^{13}$ We could have written Definition 5 to say "if $s\left[\phi_{1}\right] \cdots\left[\phi_{n}\right][\psi]$ is defined..." instead. This would be to essentially adopt a Strawson-notion of entailment, which differs from the standard notions of validity in that it requires (as a premise) that the presuppositions of the premises and conclusion are satisfied [87]. However, as an anonymous referee points out, this would yield bad results for the logic of 'therefore'. In particular, it would entail that any argument with a conclusion of the form $\therefore \phi$ is valid. For this reason, we stick with the "standard" notion of validity, which just requires the presuppositions of the premises be satisfied.
} 
example, if $s$ contains some $p$-worlds and some $\neg p$-worlds, then $s[\diamond p]=s$ and so $s[\diamond p][\neg p]=s[\neg p] \neq \varnothing$.

Dynamic entailment also differs from static entailment in its structural properties. For example, while $\diamond \phi, \neg \phi \quad \nvdash_{\mathrm{d}} \perp$, we do have $\neg \phi, \diamond \phi \vDash_{\mathrm{d}} \perp$. Thus, dynamic entailment is noncommutative (cf. [74, 85]). Moreover, dynamic entailment is nonmonotonic: while $\diamond \phi \vDash_{\mathrm{d}} \diamond \phi$, still $\diamond \phi, \neg \phi \nvdash_{\mathrm{d}} \diamond \phi$.

These two notions of entailment capture two different senses of validity that are important for different theoretical purposes. Static entailment seems to best capture a notion of validity for inferences "taken abstractly" — i.e., independent of any specific discourse. Dynamic entailment, by contrast, seems to capture a notion of validity within a discourse. On the whole, the dynamic notion seems more appropriate for modeling inferences with argument connectives such as 'therefore'. For one thing, static entailment is commutative whereas dynamic entailment is not. Yet the effect of 'therefore' on arguments seems crucially order-sensitive: $\phi, \therefore \psi$ should not, intuitively, have the same consequences as $\therefore \psi, \phi$. Moreover, as we have seen, the primary function of argument connectives like 'therefore' is inherently dynamic. It is unclear (at least conceptually) what it is to consider a sentence of the form $\therefore \phi$ as a premise "taken abstractly".

With that said, one must be careful when attempting to understand what dynamic entailment captures. For example, the following is dynamically valid:

$$
\phi \wedge \therefore \psi \vDash_{\mathrm{d}} \therefore \psi \wedge \phi .
$$

While this might seem counterintuitive at first, it is not surprising given the notion of validity that dynamic entailment is designed to capture, viz., discourse validity. Thus, the following argument as a discourse does seem valid.

(33) It's raining and therefore the streets are wet. Therefore the streets are wet and it is raining.

Though repetitive, this is essentially an argument that involves repeating the premises in the conclusion. Thus, the 'therefore' in the conclusion is not expressing the claim that 'It's raining' follows from 'The streets are wet' outright, but rather that it follows given what's been said in the discourse already.

Still, we submit that there does seem to be a sense in which inferences like $\phi \wedge \therefore$ $\psi \vDash \therefore \psi \wedge \phi$ are bad.

We can capture what is wrong with this inference using a third notion of entailment, intermediate between the static and the dynamic ones (cf. [40, §1.4]): ${ }^{14}$

Definition 6 (Kinematic Entailment, $\vDash_{\mathrm{k}}$ ) $\phi_{1}, \ldots, \phi_{n} \vDash_{\mathrm{k}} \psi$ iff for all information states $s$, if $s\left[\phi_{1}\right] \cdots\left[\phi_{n}\right]$ is defined, then $s\left[\phi_{1}\right] \cdots\left[\phi_{n}\right] \subseteq s[\psi]$.

In effect, kinematic entailment is a way of assessing how strong an update effect is. On this notion of entailment, $\phi \wedge \therefore \psi$ does not entail $\therefore \psi \wedge \phi$, since the update effect

\footnotetext{
${ }^{14}$ As with dynamic entailment, we only require the presuppositions of the premises be satisfied - that is, we do not say "if $s\left[\phi_{1}\right] \cdots\left[\phi_{n}\right]$ and $s[\psi]$ are defined...". If we had, that would validate the inference from $\phi \wedge \therefore \psi$ to $\therefore \psi \wedge \phi$, which is precisely what we are trying to avoid.
} 
of the former requires $\psi$ to be supported upon update with $\phi$, whereas the discourse effect of the latter requires that $\psi$ is already supported. So kinematic entailment gives us the resources to articulate the sense in which such an inference is bad, while preserving the idea that the primary function of 'therefore' is dynamic.

\subsection{Arguments with Non-Declarative Conclusions}

In this section, we show that our simple semantics for categorical arguments can be modified to model arguments with non-declarative conclusions (imperatives and interrogatives) (cf. Section 2.3). In order to do so, however, we need to alter our modeling of contexts as information states - as unstructured sets of worlds — and add some structure to them.

\subsubsection{Imperatives}

Let us first consider arguments with imperative conclusions (e.g., (21a-b)).

Start with the syntax. In order to add imperatives to our language, we introduce an imperative operator '!' with the following syntax.

Definition 7 (Imperative Syntax)

$$
\begin{aligned}
& \phi::=p|\neg \phi|(\phi \wedge \phi)|(\phi \wedge \therefore \phi)|(\phi \vee \phi)|(\phi \rightarrow \phi)| \square \phi \mid \diamond \phi \\
& \sigma::=\phi|\therefore \phi| ! \phi \mid \therefore ! \phi
\end{aligned}
$$

On this simple syntax, 'therefore' can only take immediate wide-scope with respect to the imperative marker. This restriction seems to be borne out, for even when 'therefore' superficially embeds under the imperative marker, the only reading that seems to be available is the wide-scope reading. For example, it seems to us that (34a) is equivalent to $(34 \mathrm{~b})$ :

(34) You can be abrasive at times...

a. Try, therefore, to be nicer.

b. $\approx$ Therefore, try to be nicer.

The second step is to settle on a semantics for imperatives. While we do not need to commit to a particular semantics for imperatives, we will show, as an example, how to adapt our semantics to a dynamic semantics for imperatives due to [76]. ${ }^{15}$ On Starr's semantics, the general idea is that imperatives express the speaker's preference for certain propositions obtaining. So, for example, 'Mow the lawn' expresses our preference for the lawn being mowed by the addressee - for the proposition that the lawn be mowed by the addressee obtaining. Starr achieves this effect by thinking of contexts as more structured than mere information states. In particular, contexts are thought of as sets of preference relations on information states. Updating with an

\footnotetext{
${ }^{15}$ For another dynamic account of imperatives, see [63, 64]. For some alternative accounts, see $[1,17,34$, 48, 70].
} 
imperative ! $\phi$ amounts to extending each preference relation with the preference that $\phi$ obtain rather than not - i.e., the result of updating the field of a preference relation with $\phi$ (in the ordinary sense) is preferable to the result of updating the field of that relation with $\neg \phi$.

Although Starr's system is only defined over the Boolean fragment of our syntax, there is a natural extension to our syntax available. Here are the formal details.

Definition 8 (Preference States) A PREFERENCE RELATION is a set $r \subseteq \wp W \times \wp W$ of pairs of sets of worlds. A PREFERENCE STATE is a set $R$ of preference relations. For each preference relation $r$, define $s_{r}=$ field $(r)$.

Definition 9 (Dynamic Semantics for Imperatives)

$$
\begin{aligned}
r[p] & =\left\{\left\langle s_{1}[p], s_{2}[p]\right\rangle \mid\left\langle s_{1}, s_{2}\right\rangle \in r \& s_{1}[p] \neq \varnothing\right\} \\
R[p] & =\{r[p] \mid r \in R\} \\
R[\phi \wedge \psi] & =R[\phi][\psi] \\
R[\phi \vee \psi] & =R[\phi] \cup R[\psi] \\
R[\phi \rightarrow \psi] & =\{r \in R \mid\{r\}[\phi][\psi]=\{r\}[\phi]\} \\
R[\square \phi] & =\{r \in R \mid\{r\}[\phi]=\{r\}\} \\
R[\diamond \phi] & =\{r \in R \mid\{r\}[\phi] \neq \varnothing\} \\
R[\therefore \phi] & = \begin{cases}R & \text { if } R[\phi]=R \\
\text { undefined } & \text { otherwise }\end{cases} \\
R[! \phi] & =\left\{r \cup\left\{\left\langle s_{r}[\phi], s_{r}[\neg \phi]\right\rangle\right\} \mid r \in R\right\}
\end{aligned}
$$

Thus, effectively, the effect of updating with $\therefore ! \phi$ is to test whether $\phi$ is ranked above $\neg \phi$ in the current preference state.

$$
R[\therefore ! \phi]= \begin{cases}R & \text { if for each } r \in R:\left\langle s_{r}[\phi], s_{r}[\neg \phi]\right\rangle \in r \\ \text { undefined } & \text { otherwise }\end{cases}
$$

This shows that the simple semantics for 'therefore' introduced in this section can easily be augmented to model arguments with imperative conclusions.

\subsubsection{Interrogatives}

Let's now turn to arguments with interrogative conclusions (e.g., (22a-b)). For the sake of simplicity, we will just focus on yes-no interrogatives (though generalization to wh-interrogatives is straightforward).

Like with imperatives, we will adopt a simplified (albeit restrictive) syntax for interrogatives. We introduce an interrogative marker '?', which scopes over propositional modal formulas but under 'therefore'.

Definition 10 (Interrogative Syntax)

$$
\begin{aligned}
& \phi::=p|\neg \phi|(\phi \wedge \phi)|(\phi \wedge \therefore \phi)|(\phi \vee \phi)|(\phi \rightarrow \phi)| \square \phi \mid \diamond \phi \\
& \sigma::=\phi|\therefore \phi| ? \phi \mid \therefore ? \phi .
\end{aligned}
$$


In order to give the semantics for interrogatives, we can piggyback on recent dynamic theories, which take the change effect potential of interrogatives to be that of raising issues. Following [30] and [2], we can model this idea by thinking of an information state, not as a set of possible worlds, but rather as a PARTITION on possible worlds - i.e., as a set of mutually disjoint but jointly exhaustive sets, or CELLS. Then the use of a yes-no interrogative might refine the partition by dividing current cells into smaller cells. More precisely, let $\pi$ be our partition on some (nonempty) subset of $W$. Updating $\pi$ with a declarative $\phi$ returns the cells in $\pi$ each updated with $\phi$, provided that the result of the update is not empty. On the other hand, updating $\pi$ with a yes-no interrogative $? \phi$ returns the union of the result of updating $\pi$ with $\phi$ and the result of updating $\pi$ with $\neg \phi$. This models the idea that yes-no interrogatives contribute by refining current partitions.

Definition 11 (Dynamic Semantics for Interrogatives)

$$
\begin{aligned}
\pi[\phi] & =\{s[\phi] \mid s \in \pi \text { and } s[\phi] \neq \varnothing\} \\
\pi[? \phi] & =\pi[\phi] \cup \pi[\neg \phi] \\
\pi[\therefore \phi] & = \begin{cases}\pi & \text { if } \pi[\phi]=\pi \\
\text { undefined } & \text { otherwise }\end{cases}
\end{aligned}
$$

The general idea is that when we use 'therefore' with an interrogative $? \phi$, all we do is to test that the issue raised by $? \phi$ is already represented in the current partition. So effectively, we are testing that adding $? \phi$ would not further refine the partition:

$$
\pi\left[\therefore ? \phi= \begin{cases}\pi & \text { if } \pi[\phi] \cup \pi[\neg \phi]=\pi \\ \text { undefined } & \text { otherwise }\end{cases}\right.
$$

An advantage of this semantics for 'therefore' with interrogatives is that it positioned to get different readings that are available for interrogative conclusions. Sometimes, we use 'therefore' with an interrogative when it is not known yet in the common ground what is a true answer to that issue raised. So, for example, we might use (22a) in a context that still does not settle the question who committed the murder. This is an inquisitive use of the interrogative. This semantics captures this use since it predicts that in this sort of context, by use of (22a), we are testing that who committed the murder is still an open issue. By contrast, suppose it is common knowledge that the only person that would stab the victim was Steve. Then, in this case, the interrogative in (22a) has the effect of a rhetorical question, which highlights that the common ground already settles the answer to that question. For in this case, adding an interrogative would not further refine a partition because the corresponding issue has been solved.

In conclusion: when stating the simple semantics for categorical arguments, in Section 3.2, we have taken information states to be simply unstructured sets of worlds. However, in order to be able to model uses of argument connectives with non-declarative conclusions in the current framework, all is needed is for more structure to be imposed on information states, so that now 'therefore' can be taken to test 
whether adding non-declarative as well as declarative conclusions alter these more structured information states.

\section{A Semantics for Complex Arguments}

The semantics presented in Section 3 nicely captures uses of 'therefore' in categorical arguments and it can be refined to capture arguments with non-declarative conclusions. However, it will not be able to model uses of 'therefore' in suppositional arguments and in complex arguments where new suppositions are introduced. So, for example, arguments of the form:

\section{Suppose $P$. Then, $Q$. Therefore, if $P$, then $Q$}

cannot be directly represented in the simple framework, as this framework does not have the tools to introduce or discharge new suppositions. Notice that we cannot simply "mimic" such complex arguments using conditionals, since this converts conditional proof into a trivial argument:

$$
P \rightarrow Q \therefore(P \rightarrow Q)
$$

It is also not straightforward to apply the simple semantics to arguments that involve parentheticals, such as (8a) and (8b). More generally, the simple framework does not have the resources to capture the anaphoric relations that argument connectives such as 'therefore' can establish in discourse.

In order to model these complexities, we introduce two main modifications to the previous framework - one syntactic and one semantic. In short: the syntactic modification is to think of discourses not as a sequences of sentences, but as sequences of labeled sentences - which track anaphoric relations in discourse. The semantic modification is to think of contexts as having a distinctive layered structure.

\subsection{Discourses and Labeled Sentences}

What is a discourse? At first, one might think the answer is simple: it is just a sequence of sentences! However, each part of a discourse bears more information than just the informational content of a sentence.

For example, in a certain part of a discourse, certain suppositions are active while others are not. But this is not indicated simply by the content of the sentence itself. Consider again (35) (repeated from (9)):

(35) Either it is raining or not. Suppose it is raining. Then better to take the umbrella. Suppose it is not raining. Then taking the umbrella will do no harm. Therefore, you should take the umbrella.

As we have seen, in (35) the final sentence 'Therefore, you should take the umbrella' could either be interpreted categorically (as in (36a)) or suppositionally (as in (36b)). 
We could more clearly represent the two readings if we "labeled" the suppositions and 'therefore' accordingly:

(36) a. CATEGORICAL: Either it is raining or not. Suppose ${ }_{1}$ it's raining. Then $_{1}$ better to take the umbrella. Suppose 2 it is not raining. Then $_{2}$ taking the umbrella will do no harm. Therefore, you should take the umbrella.

b. SUPPOSITIONAL: Either it is raining or not. Suppose ${ }_{1}$ it's raining. Then $_{1}$ better to take the umbrella. Suppose ${ }_{2}$ it is not raining. Then $_{2}$ taking the umbrella will do no harm. Therefore ${ }_{2}$, you should take the umbrella.

By themselves, however, the final sentence does not carry that information: it is only as part of a bigger discourse that it is possible to disambiguate these two different readings. This suggests when we interpret a discourse, each part of the discourse needs to represent which suppositions are active.

In addition to keeping track of which suppositions are active, we also want to be able to keep track of which suppositions are in the scope of which suppositions. For example, consider (37):

(37) Paolo is either from Bologna or from New York. Suppose ${ }_{1}$, on the one hand, that he is from Bologna. Then ${ }_{1}$, either he did his $\mathrm{PhD}$ in Bologna or he did it in the US. Suppose ${ }_{1.1}$ Paolo did his PhD in Bologna. Then $_{1.1}$, he studied Umberto Eco's work. Suppose ${ }_{1.2}$ instead Paolo did his $\mathrm{PhD}$ in the US. Then 1.2 , he must have studied philosophy of language. Therefore ${ }_{1}$, Paolo either studied semiotics or philosophy of language. Now, on the other hand, suppose ${ }_{2}$ Paolo is from New York. $\mathrm{Then}_{2}$, he did his PhD in the US. Therefore 2 , he studied philosophy of language. Either way, therefore, Paolo studied either semiotics or philosophy of language.

Here, we indicate that a supposition is in the scope of another with a decimal point. Thus, 'Suppose ${ }_{1.1}$ Paolo did his PhD in Bologna' indicates that this supposition is in the scope of the first supposition -i.e., 'Suppose ${ }_{1}$, on the one hand, that he is from Bologna.'

Moreover, the use of labels allows us to represent parenthetical remarks, which break out of the scope of a supposition, as in (38):

(38) Suppose $_{1}$ Mark went to the grocery store this morning. (Have you been? They have all sorts of exotic fruit.) Then ${ }_{1}$ he bought some dragon fruit. Therefore ${ }_{1}$, he can make an exotic fruit salad.

The fact that the parenthetical remark does not have the label ' 1 ' indicates that it is not in the scope of the supposition that Mark went to the grocery store. 
To model this formally, we will make two modifications to the syntax. First, we will add a supposition operator ' + ' to the language. Thus, our new syntax for sentences will be given as follows:

Definition 12 (Syntax for Complex Arguments)

$$
\begin{aligned}
& \phi::=p|\neg \phi|(\phi \wedge \phi)|(\phi \wedge \therefore \phi)|(\phi \vee \phi)|(\phi \rightarrow \phi)| \square \phi \mid \diamond \phi \\
& \sigma::=\phi|\therefore \phi|+\phi \mid \therefore+\phi .
\end{aligned}
$$

This syntax utilizes the same simplifying assumptions we made about the syntax of imperatives — which makes sense given that 'suppose' is an imperative. In particular, observe that we assume 'suppose' always scopes under argument connectives such as 'therefore'. This syntax captures the observation that (39a) and (39b) sound equivalent.

(39) a. It might be raining. Therefore, suppose it is....

b. It might be raining. Suppose, therefore, it is.

Second, we will think of a discourse as a sequence of what we will call labeled sentences.

Definition 13 (Labeled Sentence) A LABELED SENTENCE is a pair of the form $\langle n, \phi\rangle$, which we write as $n: \phi$ for short. Here, $n$ is a LABEL, which is a sequence of positive integers (where, for shorthand, we write $\left\langle n_{1}, n_{2}, \ldots, n_{k}\right\rangle$ in decimal form as $n_{1} . n_{2} . \cdots . n_{k}$ ) that represents which suppositions are active, and $\phi$ is a sentence (in the sense of Definition 12). Throughout, we use "0" to stand for the empty tuple \langle\rangle . Intuitively, sentences with the label 0 are asserted at the categorical level, whereas all other labeled sentences fall within the scope of some supposition.

We can represent discourses in a more intuitive format similar to Fitch proofs.

Example 14 (Proof by Cases) We can roughly formalize (35) as the following sequence of labeled sentences:

$$
0:(r \vee \neg r), 0:+r, 1: \therefore u, 0:+\neg r, 2: \therefore u, 0: \therefore u
$$

Here's an alternative Fitch-like representation of the discourse:

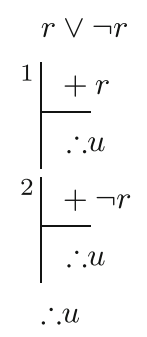


Example 15 (Nested suppositions) We can formalize (37) as follows:

$$
\begin{aligned}
& 0:(b \vee n), 0:+b, 1: \therefore\left(p h d_{b} \vee p h d_{u}\right), 1:+p h d_{b}, 1.1: \therefore u, 1:+p h d_{u} \\
& 1.2: \therefore p l, 1: \therefore(s \vee p l), 0:+n, 2: \therefore p h d_{u}, 2: \therefore p l, 0: \therefore(s \vee p l)
\end{aligned}
$$

The structure of this discourse is much easier to see in a Fitch-like representation:

$$
\begin{aligned}
& b \vee n
\end{aligned}
$$

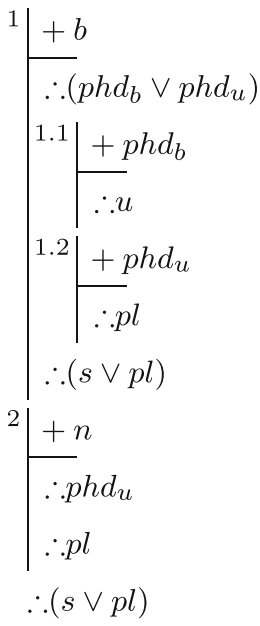

Example 16 (Parentheticals) We can formalize (38) as follows:

$$
0:+m, 0: e, 1: \therefore d, 1: \therefore f
$$

In the Fitch-like representation, parentheticals can be represented as temporary breaks within subproofs. (This is one major disanalogy between our representation of argumentative discourses and the standard Fitch-style proof system).

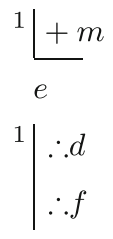

General Remarks Some general remarks about this syntax are in order. A full syntax of discourses would provide a set of necessary and sufficient conditions on discourses to be well-formed. Here, we have fallen short of giving a full set of such conditions. Overall, we take this flexibility of our system to be a virtue, since our aim here is to give a very general framework for interpreting the semantic effects of 'therefore' that can be further amended or constrained to accommodate a wide array of empirical hypotheses about which discourses are well-formed. However, we do submit that it would be natural to impose at least some constraints on acceptable discourses as a way of explaining some of the anaphoric behavior from Section 2.1. Below, we give a few examples of constraints that we find fairly plausible. 
Understanding discourses in terms of labeled sentences enables to track anaphoric relations that 'therefore' establishes in discourse. However, as we have seen (Section 2.1), an important reason for thinking that 'therefore' is an anaphoric expression is that 'therefore' needs an antecedent. It might seem as if nothing in the current syntax reflects this feature of 'therefore'. However, we can capture this anaphoric aspect of 'therefore' by imposing some additional constraints on the current syntax. One constraint is that the first labeled sentence of a discourse cannot be of the form $0: \therefore \phi$. So, (40) is not a proper discourse:

?? Therefore, we should leave.

Furthermore, a labeled sentence of the form $n: \therefore \phi$ (where $n \neq 0$ ) cannot occur unless there is a supposition prior to it that introduces the label $n$ into the discourse. This is parallel to the constraint that an anaphoric expression cannot have an index that does not appear before. In this sense, in this framework, the anaphoricity of 'therefore' is captured by the structure of discourses as laid down above together with plausible additional constraints on this discourse structure.

Another constraint on the structure of discourses might be that suppositions cannot be "idle" — i.e., introduced without a consequent (or without a discourse whose first element contains its label as an initial segment). This rules out, e.g., discourses of the form $n:+\phi, n:+\psi$, where the supposition $\phi$ is introduced but not used. Thus, a sequence of suppositions must be interpreted as introducing additional levels. To illustrate, sequences of suppositions like (41) sound marked since the second supposition is interpreted in the scope of the first (as in (41a)) rather than as a separate supposition (as in (41b)).

(41) Suppose physicalism is true. ??Suppose physicalism is false ...

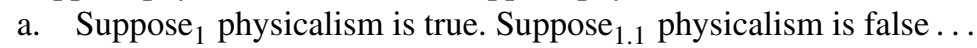

b. Suppose $_{1}$ physicalism is true. Suppose 2 physicalism is false...

Because the second supposition is interpreted within the scope of the first, as in (41a), and cannot be interpreted as in (41b), we have explained why (41) is infelicitous.

A final constraint we will mention is a prohibition on "crisscrossing" labels (cf. the "right frontier constraint" in SDRT). Essentially, this means that within a suppositional environment, we cannot refer back to other suppositions on the same level. This rules out, e.g., discourses of the form: $1: \phi_{1}, \ldots, 2: \phi_{2}, \ldots, 1: \therefore \psi$. For example, consider (42):

(42) Either it is raining or not. Suppose it is raining. Then it is wet outside.

Suppose it is not raining. Then taking an umbrella would be a hassle.

??Therefore, you should take the umbrella.

This argument seems bad no matter how you parse it. However, if 'Therefore, you should take an umbrella' is interpreted in the scope of the first supposition (that it is raining), then the argument should be fine. This shows that the last sentence cannot have the same label as the first supposition: it must either be interpreted in 
the scope of the second supposition (that it is not raining) or outside the scope of any supposition.

\subsection{Contexts as Labeled Trees}

After introducing labeled sentences, we now turn to the semantic innovation: we propose modeling contexts not as single information states, but rather as labeled trees of information states - i.e., trees where each node is given its own label. Labeled trees allow us to track multiple suppositions, nested suppositions, and anaphoric relations. ${ }^{16}$

Definition 17 (Context) A CONTEXT is a partial function $c:\left(\mathbb{N}^{+}\right)^{<\omega} \rightarrow \wp W$ from labels (i.e., sequences of positive integers) to information states. For shorthand, where $n$ is a label (i.e., a sequence of numbers), we write $c_{n}$ in place of $c(n)$. Intuitively a context is a whole tree, the nodes of which are information states. We use all the standard ancestral terms ('parent', 'child', 'descendent', and so on) that are used in talking about trees. In addition, we call the value of a context at the empty sequence 0 its CATEGORICAL STATE. We call the value of a context at a non-empty sequence a SUPPOSITIONAL STATE. Following our earlier convention, the categorical state is denoted by $c_{0}$. We will assume every context $c$ for an ordinary conversation has the following features (so that $c$ really is a labeled tree):

(1) $0 \in \operatorname{dom}(c)$

(2) if $\left\langle n_{1}, \ldots, n_{k+1}\right\rangle \in \operatorname{dom}(c)$, then $\left\langle n_{1}, \ldots, n_{k}\right\rangle \in \operatorname{dom}(c)$.

The first constraint just says the categorical state (which is the root of the tree) is always defined. The second constraint says, in effect, that a suppositional state is defined only when its parent state is defined. This rules out the possibility of "disconnected" segments of a branch.

While the meaning of a sentence is taken to be its update effect on information states (i.e., a function from information states to information states), we take the meaning of a discourse to be its update effect on a context (i.e., a function from contexts to contexts). Thus, in order to define the meaning of a discourse, we need to specify (a) how a sentence updates an information state, and (b) which information

\footnotetext{
${ }^{16}$ Our approach differs from [35] stack system in key respects. First, stacks of information states are linear whereas labeled trees include branching structure. This allows us to more perspicuously represent arguments involving multiple suppositions, such as proof by cases. Second, Kaufmann's framework does not allow one to temporarily exit a supposition, update the bottom of a stack, and then return to that supposition. This is precisely what is needed to model parentheticals, and labeled trees provide this ability. Finally, as we observe in the main text, we want to distinguish adding premises from drawing a conclusion from some premises. This distinction is blurred in Kaufmann's system. Though Kaufmann does not have a semantics for 'therefore', he does provide a semantics for 'then', but one that adds information to the stack rather than tests it. When Kaufmann's 'then' occurs under categorical premises, Kaufmann's system treats 'then' as adding information to the bottom of the stack, just like the categorical premises do. We have already noticed that taking 'therefore' (or 'then') to be informative in this way does not quite capture its functional role (Section 3.2).
} 
state a sentence in a discourse updates. To address (a), we will assume sentences update information states as in Section 3. To address (b), we make use of our definition of a discourse as a sequence of labeled sentences to tell us which information state to update at a given point in the discourse. The basic idea is simple: in effect, $n: \phi$ will tell us to update $c_{n}$ with $\phi$. However, when we introduce a new supposition in a discourse, we do not simply update the current information state with that supposition - after all, suppositions are not just assertions. Rather, we create a new information state updated with that supposition so that subsequent updates concern this new state as opposed to (say) the categorical state.

To do this, we need to give a rule for how a supposition creates a new information state. The general idea is that a new supposition effectively copies the information state of its parent and then updates that state with the supposition. Because 'suppose' does not embed under Booleans and modals, we take it to be an operator that works at the discourse level rather than at the sentential level. Its role at the discourse level is to introduce a new suppositional state - so if we think of contexts as labeled trees, it maps a labeled tree into a new one with a new branching node.

We can make these ideas more precise as follows.

Definition 18 (Adding Information) Where $n$ and $k$ are labels, we write $n \sqsubseteq k$ just in case $n$ is an initial segment of $k$ (i.e., either $k=n$ or $k$ is "above" $n$ in the labeled tree). Where $c$ is a context, let $c \uparrow_{n} \phi$ be the result of removing the worlds in $\left(c_{n}-c_{n}[\phi]\right)$ from $c_{k}$ for each $k \in \operatorname{dom}(c)$ such that $k \sqsupseteq n$ (where $c_{n}[\phi]$ is defined as in Definition 2).

In words, $c \uparrow_{n} \phi$ updates $c_{n}$ and all information states "above" $c_{n}$ in the tree with $\phi$. This allows the information added at a categorical state to percolate up to the following suppositional states. And so it will allow us to capture the idea that parentheticals introduce information at lower levels to which suppositions have then access.

Definition 19 (Introducing New Suppositions) Where $n=n_{1} \ldots n_{k}$ is a label, let $n^{+}=n_{1} \ldots . n_{k} . m$ where $m$ is the first number such that $n_{1} \ldots . n_{k} . m \notin \operatorname{dom}(c)$ (that is, $n^{+}$is the first extension of $n$ that has not been assigned an information state by $c$ ). Let $c \oplus_{n} \phi$ be just like $c$ except that we define $c_{n^{+}}=c_{n}[\phi]$.

In words, $c \oplus_{n} \phi$ is the result of extending $c$ with an additional suppositional state that is copied from $c_{n}$ and then updated with $\phi$.

Definition 20 (Dynamic Semantics for Complex Arguments) Where $\phi$ does not contain $\therefore$ or + :

$$
\begin{aligned}
c[n: \phi] & = \begin{cases}c \uparrow_{n} \phi & \text { if } c_{n} \text { is defined } \\
\text { undefined } & \text { otherwise }\end{cases} \\
c[n: \therefore \phi] & = \begin{cases}c[n: \phi] & \text { if } c_{n} \text { is defined and } c[n: \phi]_{n}=c_{n} \\
\text { undefined } & \text { otherwise }\end{cases}
\end{aligned}
$$




$$
c[n:+\phi]= \begin{cases}c \oplus_{n} \phi & \text { if } c_{n} \text { is defined } \\ \text { undefined otherwise }\end{cases}
$$

The update effect of $\therefore+\phi$ is derived from the update effects for $\therefore$ and + compositionally.

$$
\begin{aligned}
c[n: \therefore+\phi] & = \begin{cases}c[n:+\phi] & \text { if } c_{n} \text { is defined } \\
\text { undefined } & \text { otherwise }\end{cases} \\
& = \begin{cases}c \oplus_{n} \phi & \text { if } c_{n} \text { is defined } \\
\text { undefined } & \text { otherwise }\end{cases}
\end{aligned}
$$

Let's unpack these update clauses. For illustration, consider the case where $n=0$. If $\phi$ does not contain + or $\therefore$, then updating $c$ with $n: \phi$ is the result of updating $c_{0}$, as well as any suppositional states that have been defined, with $\phi$ (or, more precisely, the information contained in $\left.c_{0}[\phi]\right)$. If $n \neq 0$, then the update effect is the same, except we only update information states above $c_{n}$.

The fact that update "percolates up" a context is what allows us to account for the effects of parentheticals. Recall (38). Here, the parenthetical adds information to the categorical state (to the state antecedent to the 'Suppose Mark went...'). But it also adds information to the suppositional state created by the supposition that Mark went to the grocery store this morning. Else, the argument would not necessarily be a good one. This is captured by our update clause: when we update $c$ with $0: e$ (where $e$ stands for 'The grocery store has all sorts of exotic fruit'), we remove all $\neg e$-worlds from $c_{0}$ as well as from any suppositional state that has been defined.

If $\phi$ is of the form $+\psi$, then updating $c$ with $n:+\psi$ amounts to (i) checking whether $c_{n}$ is defined, and (ii) adding a suppositional state above $c_{n}$ that is the result of updating $c_{n}$ with $\psi$. Notice that updating with $n$ : $+\phi$ does not affect $c_{n}$ : that information state is left untouched, which is precisely what we want.

There is some question as to whether + should carry an epistemic possibility presupposition - that is, whether we should require $c[n: \phi]_{n} \neq \varnothing$ in order for $c[n:+\phi]$ to be defined. On the one hand, there is some linguistic evidence to suggest that $\ulcorner$ Suppose $\phi\urcorner$ presupposes the epistemic possibility of $\phi .{ }^{17}$ For example, the following discourse sounds strange (at least out of the blue):

(43) It is not raining. ??Suppose it is raining ...

However, discourses containing reductio reasoning do not fit this pattern. For example, the following discourse sounds perfect: ${ }^{18}$

(44) There is no largest prime number. For suppose there is ...

\footnotetext{
${ }^{17}$ This is in line with the general idea, prominent in the literature about indicative conditionals, that antecedents presuppose that the current context is compatible with them [26, 73-75].

${ }^{18}$ In dynamic semantics, it is often assumed that reducing a context to the empty set results in a "context crash". But in reductio reasoning, reducing a suppositional information state to the empty set does not necessarily result in a context crash — in fact, it is precisely what is needed! We can reconcile these two points in the following manner: context crash occurs when either the context is rendered undefined (e.g., because it fails a presuppositional test) or when the categorical state is reduced to the empty set (since that would be to rule out any world as being the actual world).
} 
The reductio example suggests that 'suppose' does not carry an epistemic presupposition after all. Our semantic clauses for + reflects this.

Finally, if $\phi$ is of the form $\therefore \psi$, then updating $c$ with $n: \therefore \psi$ amounts to checking whether (i) $c_{n}$ is defined, and (ii) $c_{n}$ supports $\psi$ (or, more precisely, whether the result of updating $c$ with $n: \psi$ does not change the information state assigned to $n$ ). If $c$ passes this test, then we continue to update $c$ with $n: \psi$. This ensures, e.g., that $n: \therefore+\psi$ adds a suppositional state with the supposition $\psi$ (as opposed to merely checking that adding that state would not crash the context, which is what would happen if the update clause simply left $c$ in tact).

\subsection{Examples}

To illustrate the semantics for discourses just introduced, let us walk through two examples.

Example 21 (Proof by Cases) Consider again (35). The effect of updating a context $c$ with this discourse is calculated as follows (throughout, let $c_{0}=s$ ):

- First, we update the categorical state $s$ with the trivial disjunction $r \vee \neg r$. So our context remains unchanged.

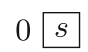

- Next, 0: $+r$ assigns $s[r]$ to the label $0^{+}=1$ (after checking that $c_{0}$ is defined, which it clearly is).

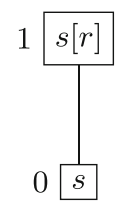

- Then 1: $\therefore u$ tests $s[r][u]=s[r]$. If it passes, it leaves the context unchanged; otherwise, the resulting context is undefined.

- Assuming $s[r]$ passes the test, 0: $+\neg r$ assigns $s[\neg r]$ to the label $0^{+}=2$ (now that $c_{1}$ is defined).

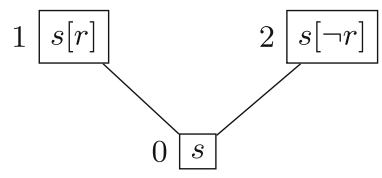

- Then 2: $\therefore u$ tests $s[\neg r][u]=s[\neg r]$. If it passes, it leaves the context unchanged; otherwise, the resulting context is undefined.

- Assuming $s[\neg r]$ passes the test, 0: $\therefore u$ tests $s[u]=s$. Since $s[r]$ and $s[\neg r]$ have passed this test, $s$ will, too. So our final context is: 


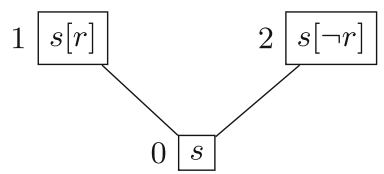

An example like (37) can be treated similarly. The only difference is that, with nested suppositions, there will be further branching at the leaves of the tree.

Example 22 (Parentheticals) Again, the goal is for parentheticals to add information to a "lower" information state and to have that information percolate "up". Indeed, our semantics achieves this. Consider (38) again. The update effect on a context $c$ with this discourse is as follows (with $c_{0}=s$ ):

- $\quad 0:+m$ maps $0^{+}=1$ to $s[m]$.

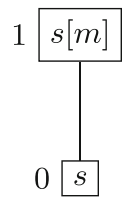

- $\quad 0$ : $e$ updates both the categorical state $s$ and the suppositional state $s[m]$ with $e$.

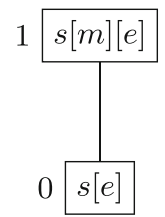

- $\quad 1: \therefore d$ tests $s[m][e][d]=s[m][e]$. Likewise for $1: \therefore f$.

Note, as it stands, we do not assume that parentheticals must always go all the way down to the categorical state. Often, this is the case but there are discourses where a parenthetical is naturally interpreted as adding information to a suppositional state. For example:

(45) Fluffy is either a cat or dog. Suppose she is a cat. Suppose further she is a Persian cat. (Arguably, she is either a Persian or a Birman.)...

With that said, if we assume that parentheticals always go down to the categorical state, we could in principle model the effects of parentheticals by adding a sentential operator. That is, we could introduce an operator $(\phi D)$ with the following update clause:

$$
c[n:(\phi)]=c[0: \phi] .
$$

\subsection{Entailment for Discourses}

Defining entailment in this framework is trickier than it might at first appear. To illustrate the complications, let us first consider dynamic entailment. As a first pass, 
we might try to define entailment as a relation between labeled sentences along the following lines:

Definition 23 (Labeled Dynamic Entailment) $n_{1}: \phi_{1}, \ldots, n_{k}: \phi_{k} \vDash_{\mathrm{d}} m: \psi$ iff for all $c$, if $c\left[n_{1}: \phi_{1}\right] \cdots\left[n_{k}: \phi_{k}\right]_{m}$ is defined, then $c\left[n_{1}: \phi_{1}\right] \cdots\left[n_{k}: \phi_{k}\right][m: \psi]_{m}=$ $c\left[n_{1}: \phi_{1}\right] \cdots\left[n_{k}: \phi_{k}\right]_{m}$.

One issue with this, however, is that defining entailment a relation between labeled sentences makes it difficult to adequately represent inference patterns that involve introducing new labels, such as conditional proof. For example, the following is not necessarily an instance of conditional proof:

$$
0:+\phi, 1: \therefore \psi \vDash 0: \therefore(\phi \rightarrow \psi)
$$

For all we know, the label 1 could have been introduced prior to supposing $\phi$. In that case, $c_{1}$ is already defined and will not necessarily be the result of updating $c_{0}$ with $\phi$; rather, $0:+\phi$ will create some new state, say $c_{2}$, and set it equal to $c_{0}[\phi]$. So in this case, 1: $\therefore \psi$ will not be testing the result of updating $c_{0}$ with $\phi$, but instead some other suppositional state.

Intuitively, the following is a way of describing an argument by conditional proof:

$$
0:+\phi, 0^{+}: \therefore \psi \vDash 0: \therefore(\phi \rightarrow \psi)
$$

That is, we want the second label to be whatever is the next extension of 0 that has not yet been introduced in the discourse. The trouble is that we do not know what that label is independent of a context. So " $0^{+}$" does not denote anything until we know what context we are looking at.

In order to describe inference patterns like conditional proof more fully, we will define entailment as a relation between (what we will call) generic labeled sentences. A generic label is either a specific label (i.e., a sequence of numbers) or the result of adding some number of "+"s to a generic label. We might define the generic labels recursively as follows.

Definition 24 (Generic Labels)

$$
\alpha::=n \mid(\alpha)^{+\cdots+}
$$

Intuitively, $(\alpha)^{+\cdots+}$ (with $m$-many $+\mathrm{s}$ ) is the result of extending $\alpha$ with the $m$ th smallest $k$ such that $\alpha . k$ has yet to be assigned an information state by our context. Note that we can iterate the $(\cdot)^{+\cdots+}$ operators to represent nested supposition labels. To simplify notation, we will drop the parentheses around the first application of this operation. So, for example, suppose $c_{1}$ is defined but not $c_{2}, c_{3}, c_{4}, \ldots$ Then, relative to $c, 0^{+}$denotes $2,0^{++}$denotes 3 , etc. By contrast, relative to $c,\left(0^{+}\right)^{+}$ denotes 2.1, rather than 3. (Note: this regiments more formally our earlier use of the same notation.) 
With these generic labels, we can now more accurately represent inference patterns that require introducing new suppositions. For example, conditional proof can be represented as the following (where $n$ is any label): ${ }^{19}$

$$
n:+\phi, \ldots, n^{+}: \therefore \psi \vDash n: \therefore(\phi \rightarrow \psi)
$$

As an application of generic labels with multiple $+s$, here is how we would represent proof by cases:

$$
n: \phi \vee \psi, n:+\phi, \ldots, n^{+}: \therefore \chi, n:+\psi, \ldots, n^{++}: \therefore \chi \vDash n: \therefore \chi
$$

Now that we have a better way of representing inference patterns, we can define entailment more precisely. Where $c$ is some context and $\alpha$ is some generic label, let $\alpha_{c}$ - i.e., the label that $\alpha$ denotes relative to $c-$ be calculated as follows:

\section{Definition 25 (Interpreting Generic Labels)}

$$
\begin{gathered}
\overbrace{(\alpha)_{c}^{+\cdots+}}^{n_{c}}=\alpha_{c} \cdot k_{m}
\end{gathered}
$$

where $\alpha_{c} \cdot k_{m}$ is the $m$ th smallest extension of $\alpha_{c}$ that has yet to be defined by $c$.

Then we can define, for instance, dynamic entailment as a relation between generic labeled sentences as follows:

Definition 26 (Generically Labeled Dynamic Entailment) $\alpha_{1}: \phi_{1}, \ldots, \alpha_{k}: \phi_{k} \vDash_{\mathrm{d}}$ $\beta: \psi$ iff for all $c$, if $c\left[\left(\alpha_{1}\right)_{c}: \phi_{1}\right] \cdots\left[\left(\alpha_{k}\right)_{c}: \phi_{k}\right]_{\beta_{c}}$ is defined, then:

$$
c\left[\left(\alpha_{1}\right)_{c}: \phi_{1}\right] \cdots\left[\left(\alpha_{k}\right)_{c}: \phi_{k}\right]\left[\beta_{c}: \psi\right]_{\beta_{c}}=c\left[\left(\alpha_{1}\right)_{c}: \phi_{1}\right] \cdots\left[\left(\alpha_{k}\right)_{c}: \phi_{k}\right]_{\beta_{c}} .
$$

Note that since every specific label is a generic label, this definition includes the first pass definition we gave above but extends it to generic labels as well.

While we take Generically Labeled Dynamic Entailment to provide a suitable dynamic notion of entailment for discourses, it is unclear how to define a corresponding static notion, even with generically labeled sentences. This is because updating

\footnotetext{
${ }^{19} \mathrm{An}$ anonymous referee points out that, at least at this level of generality, there is a difference between our formulation of conditional proof and the way it is usually formulated, e.g., in a Fitch-style natural deduction system. In the latter, the conclusion of a conditional proof involves discharging the supposition introduced ("closing" the subproof). In our formulation, the suppositional state still "hangs around" and so, without further constraints, one could conceivably go back to that suppositional state at a later point and continue reasoning from it. Our system allows us to remain neutral as to whether discharging suppositions bars a speaker from returning to it at a later point. On the other hand, even if one adopts our more general framework, one can still explain why returning to those discharged assumptions is not permitted by appealing to certain well-formedness constraints on discourses that are independently motivated (see page 22). In fact, the right-frontier constraint from SDRT would rule out exiting a suppositional state and returning to it later. So if we imposed that constraint, then there would be no material difference between conditional proof as formulated here and as formulated in a Fitch-style proof system.
} 
contexts on (generically) labeled sentences might require introducing new suppositional states. But static entailment only checks that the currently defined information state supports the sentence in question.

To illustrate, consider the following inference pattern:

$$
0:+p, 0^{+}: \therefore q \vDash 0: \therefore q
$$

Intuitively, this is not a valid inference pattern in any sense: just because $q$ is true upon supposing $p$ is true, that does not mean that $q$ is (actually, categorically) true. Yet, a static notion of entailment will have difficulties explaining this. This is because $0_{c}^{+}$is, by definition, not assigned any information state by $c$, and so $c_{0_{c}^{+}}$is undefined. Hence, trivially, if $c\left[0^{+}: \therefore q\right]_{0_{c}^{+}}=c_{0_{c}^{+}}$, then $c[0: \therefore q]_{0}=c_{0}$.

Our conclusion is that, in the presence of (generically) labeled sentences, the notion of static entailment does not make much sense. We take the lesson from this to be that there is an important difference between the validity of inferences taken in the abstract (as sequences of sentences) and the validity of inferences in discourse (as a sequence of (generically) labeled sentences or arguments). This difference validates the intuitive distinction that philosophers and cognitive scientists have independently made ([91, p. 411]; [83, pp.43-48]; [62, pp. 169-173]; [79]) between inference as an abstract entity and that of argument as essentially situated in discourse.

\section{Extensions}

\subsection{Modal Subordination}

Modal subordination bears a close resemblance to suppositional arguments, so it is natural to ask how it can be accommodated in our current framework. Consider [66] standard example:

(46) a. The wolf might come in. He will eat you.

b. Suppose a wolf comes in. He will eat you.

In (46a), we want to interpret the effect of the second sentence relative to the possibility introduced by the first modal sentence that the wolf might come in. Similarly, in (46b), the second sentence is interpreted relative to the supposition that a wolf comes in.

Or consider a similar example, involving conditionals [77, 78]:

(47) a. If a wolf comes in, we will use a gun. If we manage to shoot, we will be safe. If we bury the body, nobody will find out.

b. Suppose a wolf comes in. We will use a gun. Suppose we manage to shoot. In that case, we will be safe. Suppose we bury the body. In that case, nobody will find out.

In (47a), we want to interpret the effect of the second sentence relative to the possibility introduced by the first modal sentence that the wolf might come in. Similarly, 
in (47b), the second conditional has to be interpreted under the scope of the possibility introduced by the antecedent of the first conditional - the possibility that the wolf walks in. If this is correct, modal subordination falls under the scope of a theory of suppositional arguments.

In order to capture modal subordination, we ought to refine our entry for modals and conditionals. In particular, if we want to capture might-sentences involvement in modal subordination, their update effect should not just be that of testing the information state for whether it is compatible with their prejacent. In addition, we should model their effect as that of adding a new possibility updated with their prejacent [93, 94]. For example, in (46a), the first might-sentence should also introduce the possibility that the wolf comes in, so that the second sentence can be interpreted relative to that possibility. In our framework, that would amount to taking might-sentences to introduce a new suppositional state.

Similarly, for (47a): here the first conditional does not just test the information state for whether adding the antecedent supports the consequent and then returns that original information state. Instead, we want the conditional to also introduce a suppositional state updated with the antecedent, so that the second conditional in (47b) can be interpreted under the scope of that suppositional state. In our framework, that would amount to modeling the conditional test in a way similar to how we have modeled the result of supposing that a certain condition obtains and then testing it with 'therefore'.

Since conditionals and might-sentences have a discourse level effect, similar to the discourse level effect that our suppose operator + has, we want to capture the effect of their update at the discourse level. But because conditionals and mightsentences can embed under Boolean connectives and modals, we need to revise the update clauses slightly. Before we analyzed their semantics as updating the information states, and then we had a clause for updating on contexts that was unified for every labeled sentence that did not contain suppose + and $\therefore$ (cf. Section 3.2 , Section 4.2). In order to capture the distinctive discourse level effects of conditionals and modals, and in particular their modal subordination effects, now it is convenient to recursively assign every sentence of the language with an update effect on contexts, rather than separating out their update effects on information state and their update effects on context.

So let us start with defining the semantics for Boolean sentences in terms of context change potentials.

Definition 27 (Generalized Update for Booleans) Let $c \Uparrow_{n} s$ be the result of reassigning each $k \in \operatorname{dom}(c)$ such that $k \sqsupseteq n$ to the state $c_{k} \cap s$. That is, $c \Uparrow_{n} s$ percolates the information contained in $s$ up the tree from $n$. (Our earlier notation $c \uparrow_{n} \phi$ is essentially the special case where $c \Uparrow_{n} c_{n}[\phi]$.)

$$
\begin{aligned}
c[n: p] & =c \Uparrow_{n}\left\{w \in c_{n} \mid w(p)=1\right\} \\
c[n: \neg \phi] & =c \Uparrow_{n}\left(c_{n}-c[n: \phi]_{n}\right) \\
c[n: \phi \wedge \psi] & =c[n: \phi][n: \psi] \\
c[n: \phi \vee \psi] & =c \Uparrow_{n}\left(c[n: \phi]_{n} \cup c[n: \psi]_{n}\right)
\end{aligned}
$$


In each case, these clauses capture the idea that we update a context with a Boolean formula by performing the old update on the relevant information state (and percolating that update up the tree). According to this definition, an atomic sentence $p$ 's effect on context is to replace the state with label $n$ with the set of $p$-possible worlds in that state. The effect of the negation $\neg \phi$ is to replace the $n$-labeled state with the complement of the $n$-labeled state after updating with $\phi$. The effect of conjunction is to update the $n$-labeled state sequentially with its conjuncts. And the effect of disjunction is to replace the $n$-labeled state with the union of the updates with its disjuncts.

Now, let us turn to the modal sentences. The effect of the box is simply that of testing the $n$-labeled state:

Definition 28 (Generalized Update for 'Must')

$$
c[n: \square \phi]= \begin{cases}c & \text { if } c[n: \phi]_{n}=c_{n} \\ c \Uparrow_{n} \varnothing & \text { otherwise }\end{cases}
$$

On the other hand, the effect of the diamond is not just a testing effect. Rather, we want to capture its introducing a suppositional state (cf. [93]). The following clause tells us that the effect of updating with $n: \diamond \phi$ is to test whether (essentially) $c_{n}$ leaves open $\phi$, and, if so, then introduce a new suppositional state above $n$ that has not yet been defined (and if not, zero-out $c_{n}$ and all states above). (Here, we interpret $c \oplus_{n} \phi$ as the result of setting $c_{n^{+}}=c[n: \phi]_{n}$ where $n^{+}$is the first new extension of $n$.)

Definition 29 (Generalized Update for 'Might')

$$
c[n: \diamond \phi]= \begin{cases}c \oplus_{n} \phi & \text { if } c[n: \phi]_{n} \neq \varnothing \\ c \Uparrow_{n} \varnothing & \text { otherwise }\end{cases}
$$

These entries for $\square$ and $\diamond$ respect duality. More precisely, $n: \diamond \phi$ is equivalent to $n: \neg \square \neg \phi$, with the exception that $\diamond \phi$ introduces a new suppositional state updated with $\phi$, whereas $\neg \square \neg \phi$ does not. (The proof is left to the reader.)

This is a feature, not a bug, as it mimics the different anaphora-setting behavior of the existential quantifier and the dual of the universal one. For instance, while (48a) sounds fine, (48b) sounds odd.

(48) a. The wolf might come in. He will eat you.

b. It is not the case that the wolf must not come in. ??He will eat you.

Similarly, while (49a) sounds fine, (49b) sounds odd.

(49) a. A man came in. He had a drink.

b. It is not the case that nobody came in. ??He had a drink.

Next, consider the clause for the conditional. 
Definition 30 (Generalized Update for the Conditional)

$c[n: \phi \rightarrow \psi]= \begin{cases}c \oplus_{n} \phi & \text { if } c[n: \phi]_{n} \text { is defined and } c[n: \phi][n: \psi]_{n}=c[n: \phi]_{n} \\ c \Uparrow_{n} \varnothing & \text { otherwise }\end{cases}$

Just like $\diamond$, the conditional is a test. Whereas the $\diamond$ tests for compatibility with the information state, $\rightarrow$ tests for support of the consequent by a suppositional state which is introduced by the antecedent. It differs from other test semantics for conditionals [26, 74, 84] in that if the test is positive, it returns not just the original context prior to update with the antecedent but the context with a new suppositional state updated with the antecedent (though see [75] for a proposal similar to the one outlined here, motivated by an analysis of Sobel sequences). This is crucial to our account of modal subordination with conditionals.

In general, one conditional is modally subordinated to another if the former has a label that was introduced by the latter. ${ }^{20}$ An important element of our account is that which sentences are modally subordinated to which sentences is represented by the labels: either the subordinated conditionals have the same label or they have an incremental label. So in the following example, the second is modally subordinated to the first, and, moreover, the third is modally subordinated to the second:

$$
\begin{aligned}
& n: A \rightarrow B, n^{+}: C \rightarrow D,\left(n^{+}\right)^{+}: E \rightarrow F
\end{aligned}
$$

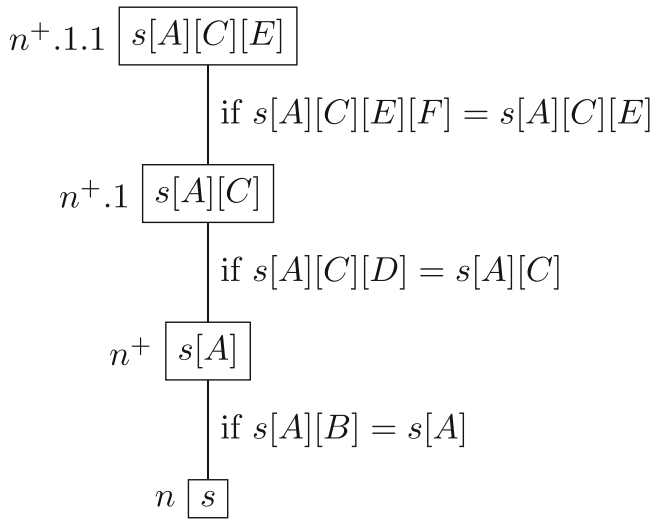

In Section 4.4, we have seen how to formalize the $\left(n^{+}\right)^{+}$notation. Consistently with that formalization, we use $\left(n^{+}\right)^{+}$for the label $n . k .1$, where $n . k=n^{+}$is the label introduced by the first conditional (since $n . k$ is new, the first undefined proper extension of $n . k$ is $n . k .1$ ). By contrast, in the following example, the second and third conditionals are both modally subordinated to the first, but the third conditional is

\footnotetext{
${ }^{20}$ Our account of modal subordination significantly differs from [77] in that (1) we adopt a test semantics for the conditional and (2) we situate the background information state as part of a tree-like structure from the context. Notably, our account is able to address some of the worries that [78, p. 405] raises for dynamic theories of content, specifically concerning whether they are capable of modeling modal subordination for conditionals.
} 
not modally subordinated to the second (meaning the second conditional's antecedent has been discharged):

$$
\begin{aligned}
& n: A \rightarrow B, n^{+}: C \rightarrow D, n^{+}: E \rightarrow F \\
& \text { if } s[A][C][D]=s[A][C]=n^{+} .1 s[A][C]=s[A][E]
\end{aligned}
$$

Here, the fact that we are using the same label for both the second and third conditionals indicates that they are both testing the suppositional state introduced by the first, but the third conditional is not within the scope of the second (for that interpretation, we would need to use $\left(n^{+}\right)^{+}$for third conditional's label). For example, consider (50):

(50) If a wolf comes in, we will use a gun. If we manage to shoot we will be safe. If we bury the body nobody will find out.

In (50), each conditional is modally subordinated to the previous one. So it is like the first case above, where the third conditional has a label of the form $\left(n^{+}\right)^{+}$. On the other hand, in (51), the final conditional is only modally subordinated to the first one, but not to the second, on pain of inconsistency:

(51) If a wolf comes in, we will use a gun. If we manage to shoot we will be safe. If we do not manage to shoot it, we will be in trouble.

So this example is more like the second case, where the third conditional has a label of the form $n^{+}$.

Like with $\diamond, \rightarrow$ does not introduce a new suppositional state when embedded under negation. (The proof is left to the reader.) This is to be expected, as illustrated by (52):

(52) It is not the case that if a wolf walks in, we won't use a tranquilizer gun. ??If we managed to shoot it, we will be safe.

Finally, the clauses for $\therefore$ and + are as they were before:

Definition 31 (Generalized Update for 'Therefore' and 'Suppose')

$$
\begin{aligned}
c[n: \therefore \phi] & = \begin{cases}c[n: \phi] & \text { if } c_{n} \text { is defined and } c[n: \phi]_{n}=c_{n} \\
\text { undefined } & \text { otherwise }\end{cases} \\
c[n:+\phi] & = \begin{cases}c \oplus_{n} \phi & \text { if } c_{n} \text { is defined } \\
\text { undefined } & \text { otherwise }\end{cases}
\end{aligned}
$$


Except when $\phi$ is a conditional, a might-sentence, or a supposition, updating $c$ with $n: \therefore \phi$ will be redundant (i.e., return $c$ ) if it passes the test. Updating with $n: \therefore(\phi \rightarrow \psi), n: \therefore \diamond \phi$, or $n: \therefore+\phi$ is, in a sense, uninformative in that $c_{n}$ will remain the same post-update (assuming $c$ passes the test). But it will not be a redundant update on the context in that it does have the effect of changing the context by introducing new suppositional states.

\subsection{Flavors of 'therefore'}

As we have seen in Section 3.1, 'therefore' can be used with different flavors: not only logical/deductive but also causal flavor (e.g., (28a), (28b)) as well as practical (e.g., (29)). It is natural to wonder how the dynamic account developed in this paper can account for possibility of arguments with different flavors.

To begin with, consider again (28b). To the extent to which this argument sounds like a good one, it does seem to involve an unstated assumption - the causal law that whenever a cigarette is dropped in a pile of hay, the hay will catch fire. On this analysis, when used in causal arguments, 'therefore' is an invitation to test that a state $s$ supporting the causal laws $C$ and augmented with the premises supports the conclusion (i.e. to test that $s[C]\left[P_{1}\right] \cdots\left[P_{n}\right]$ supports $Q$ ). This way of thinking of causal arguments enables us to model the fact that, e.g., once an agent has learnt that whenever a cigarette is dropped in a pile of hay, the hay will catch fire, they will be able to draw the conclusion that 'therefore' this pile of hay caught fire, once they learn that in some specific case a cigarette was dropped in a pile of hay. Similar considerations hold for arguments with practical flavor, such as (29). Here the unstated assumption might be a moral or prudential law, or even a rule of etiquette.

So our analysis can easily accommodate both causal and practical arguments. ${ }^{21}$ One might now wonder, however, about inductive or abductive arguments. In such arguments we really do seem to jump to conclusions that are not supported by our current information state, so in these cases one might worry that our test semantics for 'therefore' cannot possibly work.

\footnotetext{
${ }^{21}$ There are different ways of implementing this general proposal. One is to think of these arguments as enthymematic — as missing a premise (causal laws, moral laws, etc). A different approach would be to treat 'therefore' as a modal like 'must' on a Kratzerian analysis [41, 42] and to extend this Kratzerian analysis to our dynamic framework. On a Kratzerian analysis, different possible flavors of 'must' are modeled in a unified fashion by a function (the 'modal base') that takes a designated possible world into a set of propositions that varies depending on the flavor of the modal: it can be the set of evidence available to one if the interpretation of the modal is epistemic, or the set of deontic laws if the interpretation of the modal is deontic, and so on. The same analysis can be extended to capture different flavors of 'therefore'. We could import this idea, proposed by [15, pp. 295-6], into our current framework but if we did, we would need to make some adjustments to the proposal, since currently, in our semantic clauses for 'therefore' (Sections 3.2 and 4.2), there is no designated world that could be the input of the modal base function. This could be obviated by taking 'therefore' to test the modal base for each possible world in the relevant state. If the test fails for any world in the state, then the resulting state is undefined. This different approach would have to augment the current formal framework with an accessibility relation.
} 
To begin with, notice, however, that often in inductive and abductive arguments, we use 'therefore' with a qualified conclusion, as in (53a-b):

(53) a. The sun has risen every day in the past. Therefore, it seems safe to assume that it will rise again tomorrow.

b. The sun has risen every day in the past. Therefore, probably it will rise again tomorrow.

Consider (53a). It is a good argument also because the conclusion that the sun will rise again tomorrow is qualified by 'it is safe to assume...' . Similarly for (53b), where the qualification is done by the adverb 'probably'. These inductive arguments with qualified conclusions can be accommodated by our analysis, just by adding further information or structure to our states. For example, provided that the state is given probabilistic structure (as in [95]), it seems plausible that when augmented with the premises of $(53 \mathrm{~b})$, the state does support that it is probable that the sun will rise tomorrow, as in (53b).

The question now arises: Can 'therefore' ever be felicitously used in genuinely inductive and abductive arguments without qualifications of sort? We report that many speakers do endorse arguments such as (54a) and (54b) as sound:

(54) a. Mark owns a Bentley. Therefore, he must be rich. [50]

b. The sun has risen every day in the past. Therefore, the sun will rise again tomorrow.

However, at closer look, it is not entirely clear that these conclusions are not also qualified in the relevant sense. (54a) involves the modal 'must', that at least some think to be non-factive [23, 47]. If 'must' is not factive, the conclusion in (54a) does not entail that Mark is rich, but only that it is very likely given our evidence that Mark is rich [22]. In that case, we can analyze the conclusion of (54a) in a way similar to other probabilistically qualified conclusions.

Even the conclusion of (54b) may be said to be qualified by the use of 'will'. For one, it is plausible that 'will' has "epistemic" uses, as illustrated in (55) (cf. [16, 20, 21, 38]).

(55) John will be in London by now.

In the case of future-oriented uses, it is hard to tell the epistemic and non-epistemic readings apart, but the availability of epistemic readings for sentences such as (55) at least calls into question whether the conclusion of (54b) is really unqualified. Moreover, as [56] observes, the use of 'will' is often licensed even in cases where the conclusion is only probabilistically supported, as in (56).

(56) [Looking at the weather app, which says there's a 99\% chance of rain

tomorrow.] It will rain tomorrow.

So on plausible views of modals such as 'must' and 'will', in both (54a) and (54b), the conclusion is not obviously "unqualified".

We will not be able to settle here the thorny issue of whether 'therefore' can ever be used in inductive and abductive arguments with non-qualified conclusions. For 
example, there is disagreement about whether the following (not involving modals, or even tense particles in the conclusion) is felicitous or not:

?Mark owns a Bentley. Therefore, he is rich.

We would like to conclude by mentioning a possible analysis of inductive arguments which does predict that uses of 'therefore' in inductive arguments with non-qualified conclusions are possible.

On this proposal inductive arguments are also treated as involving an unstated assumption, as our earlier proposal for causal and practical arguments. In the case of inductive arguments, however, the unstated assumption is the so-called principle of uniformity of nature, or a specific form thereof. The principle of uniformity of nature states that the course of nature continues uniformly the same, and in particular that the uniformity observed in the past will hold for the present and future as well. An inductive argument might be thought to be involving the unstated assumption that the course of nature, in the respects specified by the premises, continues uniformly as the same. By thinking of inductive arguments in this fashion, we allow for the possibility that 'therefore' can be sometimes used in genuine inductive arguments with nonqualified conclusions, since in these arguments, 'therefore' would be testing that the state with the premises and this unstated assumption support the conclusion. For example, in (54b), the conclusion that the sun will rise again tomorrow is supported by a state augmented with the premise that the sun has risen every day in the past and the unstated assumption that the course of nature continues uniformly as the same, with respect to sun rising.

This approach might put us in position to explain the distinctive failure of monotonicity that we observe with inductive arguments. While our dynamic notion of entailment is non-monotonic as we have seen (Section 3.3), its non-monotonicity is isolated to arguments with epistemic modals in the conclusion: arguments without epistemic modals in the conclusion are monotonic. By contrast, inductive arguments exhibit a distinctive kind of non-monotonocity that does not involve epistemic modals (at least, assuming 'will' does not count as a qualifier in the relevant sense). So for example, consider:
a. [(54b)] The sun has risen every day in the past. Therefore, the sun will rise again tomorrow.
b. The sun has risen every day in the past. And today is the end of the world. ??Therefore, the sun will rise again tomorrow.

On the current analysis, (58a) is a good argument only on the unstated assumption that the course of nature will continue as before in the respects specified by the premises of (58a) (i.e., with respect to the sun rising). And even if (58a) is a good argument, (58b) does not need to be a good argument, since a different instance of the principle of uniformity of nature is invoked - viz., that the course of nature will continue as before in the respects specified by the premises of (58b) (which include the premise that today is the end of the world). And from this principle, together with the premises of $(58 \mathrm{~b})$, the conclusion that the sun will rise again does not follow. Put differently: the principle of uniformity of nature says the future will resemble/be similar to the past in the respects specified by the premises. By including further premises 
in the argument, we change the notion of similarity that is invoked by the unstated assumption.

To sum up, we outlined an analysis of causal and practical uses of 'therefore' that strikes us as promising. In inductive and abductive cases, it is less clear that use of 'therefore' is licensed when the conclusions are not appropriately qualified. We have nonetheless sketched a possible analysis of uses of 'therefore' in inductive arguments, that extends to account for distinctive failures of monotonicity that are typical for inductive arguments.

\section{Conclusions and Outstanding Issues}

In this paper, we have provided a comprehensive semantics of arguments as used in ordinary discourse. The framework that we have developed takes contexts to be more structured than usually understood in dynamic semantics. Instead of them being information states, or information states with some imbued structure, we proposed that the sort of context that is needed to model complex arguments, as well as suppositional arguments and the anaphoric relations that those establish in discourse, ought to have a distinctive layered structure. As we have shown along the way, this structure can be visualized as a tree, whose branches model novel suppositions and whose nodes are information states. Alternatively, we can represent these tree-like structures in a linear fashion - e.g., as having something like the form of a Fitch proof. In effect, our approach can be seen as a dynamic implementation of Fitch proofs of this sort (but with additional flexibility in that one can temporarily exit suppositional environments, as in parentheticals).

Interestingly, the structure of contexts that we uncovered turns out to be, in some respects, a simplified application of the structure of contexts proposed by SDRT (e.g., $[7,36,46])$. In SDRT, labels are attached to discourse representation structures, discourse coherence relations, and Boolean combinations thereof. Our framework turns out to be a "simplified" application of SDRT in that, unlike regular SDRT, we did not build in discourse coherence relations into our models of contexts and we just focused on argumentative discourses. In some ways, the similarities are unsurprising, since (as we saw in Section 2.1), argument connectives exhibit distinctively anaphoric behavior. So, one would expect a leading dynamic theory of anaphora to be applicable to such expressions. On the other hand, as we mentioned in Section 2.1, there are a number of significant differences between pronominal anaphora and the kind of propositional anaphora exhibited by 'therefore'. And there has not been much discussion of the anaphoric behavior of argument connectives within the literature on SDRT, nor have their uses in proof-like discourses and complex arguments, such as reductio and conditional proofs, been studied. Our theory in this paper demonstrates that it is at least possible to apply an SDRT-like framework towards this distinctive kind of anaphora, which in turn lends further support to the fruitfulness of the framework. But more work is needed to determine how our theory of argument connectives interacts with other parts of standard SDRT. 
We will conclude by listing some notable questions for further investigation.

Anaphoric Structure Our analysis presupposes that discourses come to us with a settled anaphoric structure and we are assuming that as semanticists, we can interpret discourses as given to us with such a structure. This is analogous to the situation we find ourselves in with pronominal anaphora: semanticists studying anaphora only concern themselves with the semantics of sentences with anaphoric relations built in, not with how exactly -i.e., through which mechanisms of relevance, discourse resolutions, etc. - those anaphoric relations are determined in context. While a sizable literature concerns how it is that a discourse comes to have these anaphoric relations -i.e., what are the mechanisms of anaphora resolutions (e.g., [37, 67, 69, 82, 86]) - more work is there to be done on whether and how these mechanisms differ for pronominal anaphora and for the sort of (propositional) anaphora that we observe with 'therefore'.

Argument Connectives and Performatives In Section 3.2.1, we have observed some similarities between 'therefore' and performatives such as 'I hereby conclude'. The analogy with performatives is fitting, for using 'therefore' does amount to doing something -i.e., drawing a conclusion. However, while 'therefore' does resemble performatives such as 'I hereby conclude' in some of its syntactic distribution, some differences are striking. One difference is that they do not mean the same under third-person attitude reports:

(59) a. Mary believes that Paolo is from Turin and, therefore, Italian.

b. ??Mary believes that Paolo is from Turin and, I conclude, Italian.

Another difference is that, as noted (footnote 8), the phenomenon of denegation observed for performatives [31, 71] does not seem to concern also 'therefore'. All in all, more work is needed to figure out the analogies and disanalogies between 'therefore' and performatives.

Differences Among Argument Connectives In this paper, we have treated argument connectives such as 'therefore', 'thus', 'then', 'so', etc. in the same way, formalizing them all as $\therefore$ (see Section 3.2.1). While it is true that 'therefore', 'hence', and 'thus' exhibit similar behavior, the cases of 'then' and 'so' are different, as they appear to have different distribution properties. While 'then' felicitously embeds under conditionals, 'therefore' does not (or at least, is less preferred), as illustrated in (60) (cf. [59]).

(60) a. If it is raining, then the streets are wet.

b. ?If it is raining, therefore the streets are wet.

By contrast, in categorical contexts, 'therefore' is preferred to 'then', as illustrated in (61):

(61) It is raining.

a. Therefore, the street are wet.

b. ?Then the street are wet. 
Next, consider 'so'. When used as an argument connective, 'so' seems to be more flexible in its distribution properties than 'therefore': the former can be used in categorical or suppositional reasoning alike. Also, 'so' can be used without linguistic antecedents, as in 'So, you have arrived!' and in this respect seems to exhibit a deictic behavior. Moreover, while one cannot easily mix 'therefore', 'thus', or 'hence', one can use 'so' and 'therefore' together, as in:

(62) Mary is a progressive.

a. So, she therefore supports the Green New Deal.

b. $\approx$ So therefore, she supports the Green New Deal.

A complete theory of the semantics of argument connectives ought to be sensitive to these distributional differences. Another potential extension is a study of the semantics of other argument connectives like 'arguably', 'presumably', 'normally', and so forth. On the one hand, these phrases seem to play a similar role to 'therefore'. On the other, they do not seem as strict in terms of requiring that the conclusion strictly follows from the premises. These phrases can also interact with 'therefore' and that deserves further investigation than we are capable of providing here. Finally, the analogies between 'therefore' and causal connectives such as 'since' and 'because' — often emphasized by discourse coherence theorists (cf. [6, 7, 12-14, 32, 36]) is worth a closer study than we have provided here.

Arguments and Justification Given the connection of our framework to the Fitch proof system, it is natural to wonder how one might represent the justifications of steps in a Fitch proof. In a Fitch proof, one must cite introduction/elimination rules to justify each step. In ordinary discourse, this can manifest with locutions like "by...", as in:

(63) It's raining. Therefore, by disjunction introduction, it's either raining or snowing.

As it stands, our dynamic semantics does not test for the justification of an inference; it only tests whether the conclusion is supported by the premises given the context. One interesting area of future research is to explore ways of including justifications within arguments. For this purpose, one possible route is to introduce a kind of justification logic into a dynamic setting. Justification logic is a variant of epistemic logic that introduces sentences paired with a justification - so sentences have the form $t: \phi$ where $t$ is a term for a justification [3-5, 25]. Combining our dynamic framework with justification logic $[3-5,25]$ is a promising way to model the effect of justification phrases like 'by...'.

Incorporating justifications into the current framework also has another potential application. In this paper, we have not discussed zero-premise arguments, that are quite central to natural logic systems as well as to mathematical argumentative practices. Our omission is partly due to the fact we have focused on arguments that are formulated with argument connectives, like 'therefore', which as we have seen, require an antecedent (Section 2.1). That does not mean, however, that zero-premise 
arguments cannot be made in ordinary discourse. We think it is plausible that an utterance of 'Either it is raining or it is not raining' could, for example, be thought of as a zero-premise argument, in which we assert the conclusion to hold "by logic", so to say. This suggests that zero-premise arguments can be modeled as cases where an assertion is given a special kind of justification offered by the entire logical system, rather than by a specific rule (cf. [60]).

Embeddings, Subjunctive Arguments, Pragmatics Further areas of further research encompass uses of 'therefore' under embeddings such as belief reports and epistemic modals (Section 2.2), as well as the study of subjunctive arguments, which we have to leave out. Finally, while we have primarily focused on the semantics of arguments, there is a lot of interesting work to be done on the pragmatics of arguments. For example, one outstanding question here is how arguments, as speech acts, differ from other related speech acts such as explanations (cf. [61]). Another question is what role relevance plays in assessing arguments - e.g., is relevance to be captured by the semantics of argument connectives or is it best captured by pragmatics?

A full theory of arguments will have to address these questions at some point. Here, we have only provided a starting framework for addressing them, as well as the motivations for thinking that they are worth asking.

Acknowledgements We would like to thank the participants at Cornell Interdisciplinary Semantics Reading Group, Cornell Language Workshop, and the Munich Center for Mathematical Philosophy Colloquium for their comments. Special thanks go to Daniel Altshuler, Ash Asudeh, Ivano Ciardelli, Guillermo Del Pinal, Hannes Leitgeb, Matt Mandelkern, Sarah Murray, Mats Rooth, Julian Schlöder, Will Starr, an anonymous referee and the editors for suggestions that have improved the paper. We are particularly grateful to Frank Veltman for encouragement and advice on this project.

\section{References}

1. Aloni, M. (2007). Free choice, modals, and imperatives. Natural Language Semantics, 15(1), 65-94.

2. Aloni, M., Beaver, D., Clark, B., et al. (2007). The dynamics of topic and focus. In Questions in dynamic semantics, chap. 6 (pp. 123-145). Brill.

3. Artemov, S. (1995). Operational modal logic. Tech. Rep MSI 95-29. Mathematical Sciences Institute, Cornell University.

4. Artemov, S., \& Fitting, M. (2019). Justification logic: reasoning with reasons Vol. 216. Cambridge: Cambridge University Press.

5. Artemov, S. N. (2008). The logic of justification. Review of Symbolic Logic, 1(4), 477-513.

6. Asher, N. (1993). Reference to abstract entities. Dordrecht: Kluwer Academic Publishers.

7. Asher, N., \& Lascarides, A. (2003). Logics of conversation. Cambridge: Cambridge University Press.

8. Bach, K. (1999). The myth of conventional implicature. Linguistics and Philosophy, 22(4), 327-366.

9. Beaver, D. (1999). Presupposition accommodation: A plea for common sense. Logic, Language and Computation, 2, 21-44.

10. Beaver, D. (2001). Presupposition and assertion in dynamic semantics Vol. 29. Stanford: CSLI publications.

11. Bittner, M. (2014). Temporality: universals and variation. Hoboken: Wiley-Blackwell.

12. Bras, M., Le Draoulec, A., \& Vieu, L. (2001). French adverbial 'puis' between temporal structure and discourse structure. Semantic and pragmatic issues in discourse and dialogue: experimenting with current theories. CRiSPI series, 9, 109-146.

13. Bras, M., Le Draoulec, A., \& Vieu, L. (2001). Temporal information and discourse relations in narratives: the role of French connectives 'puis' and 'un peu plus tard'. In Proceedings of the workshop 
on temporal and spatial information processing, (Vol. 13 p. 7). Association for Computational Linguistics.

14. Bras, M., Le Draoulec, A., \& Asher, N. (2009). A formal analysis of the French temporal connective 'alors'. Oslo Studies in Language 1(1), 149-170.

15. Brasoveanu, A. (2007). Structured nominal and modal reference. $\mathrm{PhD}$ thesis, Rutgers University New Brunswick, NJ.

16. Cariani, F., \& Santorio, P. (2018). Will done better: Selection semantics, future credence, and indeterminacy. Mind, 127(505), 129-165.

17. Charlow, N. (2014). Logic and semantics for imperatives. Journal of Philosophical Logic, 43(4), 617-664.

18. Chomsky, N. (1981). Lectures on Government and Binding: The Pisa Lectures. 9, Walter de Gruyter.

19. Chomsky, N. (1982). Some concepts and consequences of the theory of government and binding. Cambridge: MIT Press.

20. Condoravdi, C. (2001). Temporal interpretation of modals-modals for the present and for the past. In The construction of meaning. Citeseer.

21. Condoravdi, C., \& Deo, A. (2008). Aspect shifts in indo-aryan. In Proceedings of the 18th International Congress of Linguistics (pp. 1-20). Seoul, Korea.

22. Del Pinal, G. (2021). Probabilistic semantics for epistemic modals: normality assumptions, conditional epistemic spaces, and the strength of 'must' and 'might'. Linguistics and Philosophy.

23. Del Pinal, G., \& Waldon, B. (2019). Modals under epistemic tension. Natural Language Semantics 27(2), 135-188.

24. Elswyk, V. P. (2019). Propositional anaphora. Philosophical Studies, 176(4), 1055-1075.

25. Fitting, M. (2005). The logic of proofs, semantically. Annals of Pure and Applied Logic, 132(1), 1-25.

26. Gillies, A. (2009). On truth-conditions for if (but not quite only if). Philosophical Review, 118(3), 325-349.

27. Gillies, A. (2010). Iffiness. Semantics and Pragmatics, 3, 4-1.

28. Goldstein, S. (2019). Generalized update semantics. Mind, 128(511), 795-835.

29. Grice, H. P. (1975). Logic and Conversation. In P. Cole, \& J. Morgan (Eds.) Syntax and semantics, (Vol. 3 pp. 43-58). New York: Academic Press.

30. Groenendijk, J. et al. (2003). Questions and answers: Semantics and logic. In Proceedings of the 2 nd CologNET-ElsET symposium. Questions and answers: theoretical and applied perspectives (pp. 1623). The Netherlands: Universiteit Utrecht Utretcht.

31. Hare, R. M. (1970). Meaning and speech acts. The Philosophical Review, 79(1), 3-24.

32. Hobbs, J. R. (1985). On the coherence and structure of discourse. CA: CSLI Stanford.

33. Karttunen, L., \& Peters, S. (1979). Conventional lmplicature. In Presupposition (pp. 1-56). Brill.

34. Kaufmann, M. (2011). Interpreting imperatives Vol. 88. Berlin: Springer Science \& Business Media.

35. Kaufmann, S. (2000). Dynamic context management. Formalizing the dynamics of information, pp. 171-188.

36. Kehler, A. (2002). Coherence, reference and the theory of grammar. CA: CSLI Publications Stanford.

37. Khalid, B., Alikhani, M., Fellner, M., et al. (2020). Discourse coherence, reference grounding and goal oriented dialogue. arXiv:200704428.

38. Khoo, J. (2015). On indicative and subjunctive conditionals. Philosophers' Imprint 15.

39. King, J. C., \& Lewis, K. S. (2018). Anaphora. In E. N. Zalta (Ed.) The Stanford Encyclopedia of Philosophy, fall 2018 edn. Metaphysics Research Lab, Stanford University.

40. Kocurek, A. W. (2018). What can you say? Measuring the expressive power of languages. PhD thesis, UC Berkeley.

41. Kratzer, A. (1977). What 'must' and 'can' must and can mean. Linguistics and Philosophy, 1(3), 337-355.

42. Kratzer, A. (1981). The notional category of modality. Words, Worlds, and Contexts: New Approaches in Word Semantics, 6, 38.

43. Krifka, M. (2013). Response particles as propositional anaphors. In Semantics and linguistic theory (pp. 1-18).

44. Krifka, M. (2014). Embedding illocutionary acts. In Recursion: Complexity in cognition (pp. 59-87). Springer.

45. Krzyżanowska, K., Wenmackers, S., \& Douven, I. (2013). Inferential conditionals and evidentiality. Journal of Logic, Language and Information, 22(3), 315-334. 
46. Lascarides, A., \& Asher, N. (2007). Segmented discourse representation theory: Dynamic semantics with discourse structure. In H. Bunt, \& R. Muskens (Eds.) Computing meaning (pp. 87-124). Netherlands, Dordrecht: Springer.

47. Lassiter, D. (2016). Must, knowledge, and (in) directness. Natural Language Semantics, 24(2), 117-163.

48. Lewis, D. (1972). General semantics. In Semantics of natural language (pp. 169-218). Springer.

49. Lewis, D. (1979). Scorekeeping in a language game. In Semantics from different points of view (pp. 172-187). Springer.

50. Lust, B., Solan, L., Flynn, S., et al. (1986). A comparison of null and pronoun anaphora in first language acquisition. In Studies in the acquisition of anaphora (pp. 245-277). Springer.

51. Mandelkern, M. (2016). Dissatisfaction theory. In Semantics and Linguistic Theory (pp. 391-416).

52. Mandelkern, M. (2019). What 'must' adds. Linguistics and Philosophy, 42(3), 225-266.

53. Murray, S. (2014). Varieties of update. Semantics and Pragmatics, 7, 2-1.

54. Needham, S. M. (2012). Propositional anaphora in english: The relationship between so and discourse. $\mathrm{PhD}$ thesis, Carleton University.

55. Neta, R. (2013). What is an inference? Philosophical Issues, 23(1), 388-407.

56. Ninan, D. (2021). Assertion, evidence and the future. Ms, Tufts University.

57. Parsons, J. (2011). Cognitivism about imperatives. Analysis, 72(1), 49-54.

58. Parsons, J. (2013). Command and consequence. Philosophical Studies, 164(1), 61-92.

59. Pavese, C. (2017). On the meaning of "therefore". Analysis, 77(1), 88-97.

60. Pavese, C. (2021). Lewis Carroll's regress and the presuppositional structure of arguments. Linguistics and Philosophy. https://doi.org/10.1007/s10988-020-09320-9.

61. Pavese, C. (forthcoming). The semantics and pragmatics of argumentation. In D. Altshuler (Ed.) Linguistics meets philosophy. Cambridge: Cambridge University Press.

62. Pinto, R. (2001). The relation of argument to inference. In Argument, Inference and Dialectic (pp. 3245). Springer.

63. Portner, P. (2004). The semantics of imperatives within a theory of clause types. In Semantics and linguistic theory (pp. 235-252).

64. Portner, P. (2007). Imperatives and modals. Natural Language Semantics, 15(4), 351-383.

65. Potts, C. et al. (2005). The logic of conventional implicatures Vol. 7. Oxford: Oxford University Press.

66. Roberts, C. (1989). Modal subordination and pronominal anaphora in discourse. Linguistics and Philosophy, 12(6), 683-721.

67. Roberts, C. (1998). The place of centering in a general theory of anaphora resolution. Centering Theory in Discourse, pp. 359-400.

68. Ross, H. (1984). Inner islands. In Annual meeting of the berkeley linguistics society (pp. 258-265).

69. Sag, I. A., \& Hankamer, J. (1984). Toward a theory of anaphoric processing. Linguistics and Philosophy, pp. 325-345.

70. Schwager, M. (2006). Conditionalized imperatives. In Semantics and linguistic theory (pp. 241-258).

71. Searle, J. R. (1969). Speech acts: An essay in the philosophy of language Vol. 626. Cambridge: Cambridge University Press.

72. Stalnaker, R. (1973). Presuppositions. Journal of Philosophical Logic, 2(4), 447-457.

73. Stalnaker, R. (1976). Indicative conditionals. In Ifs (pp. 193-210). Springer.

74. Starr, W. (2014). A uniform theory of conditionals. Journal of Philosophical Logic, 43(6), 1019-1064.

75. Starr, W. (2014). What 'If'? Philosophers' Imprint, 14(10).

76. Starr, W. (2020). A preference semantics for imperatives. Semantics and Pragmatics, 13, 6.

77. Stojnić, U. (2017). One's modus ponens: Modality, coherence and logic. Philosophy and Phenomenological Research, 95(1), 167-214.

78. Stojnić, U. (2019). Content in a dynamic context. Nô̂s, 53(2), 394-432.

79. Stojnić, U. (forthcoming). Anatomy of arguments in natural language discourse. Oxford Studies in Philosophy of Language.

80. Stokke, A. (2017). Conventional implicature, presupposition, and lying. Aristotelian Society Supplementary, 91(1), 127-147.

81. Stone, M. (1994). The reference argument of epistemic must. In Proceedings of IWCS (pp. 181-190).

82. Stone, M., \& Lascarides, A. (2010). Coherence and rationality in grounding. In Proceedings of the 14th workshop on the semantics and pragmatics of dialogue (pp. 51-58). Poznán Poland.

83. van Eemeren, F. H., \& Grootendorst, R. (1984). Speech acts in argumentative discussions: A theoretical model for the analysis of discussions directed towards solving conflicts of opinion. Studies of argumentation in pragmatics and discourse analysis, 1. 
84. Veltman, F. (1985). Logics for conditionals. PhD thesis, University of Amsterdam.

85. Veltman, F. (1996). Defaults in update semantics. Journal of Philosophical Logic, 25(3), 221-261.

86. Venditti, J., Stone, M., Nanda, P., et al. (2002). Discourse constraints on the interpretation of nuclearaccented pronouns. In Speech Prosody 2002, International Conference.

87. von Fintel, K. (2001). Counterfactuals in a dynamic context. Current Studies in Linguistics Series, 36, 123-152.

88. von Fintel, K. (2008). What is presupposition accommodation, again? Philosophical Perspectives, 22, $137-170$.

89. von Fintel, K., \& Gillies, A. (2007). An opinionated guide to epistemic modality. Oxford Studies in Epistemology, 2, 32-62.

90. von Fintel, K., \& Gillies, A. (2010). Must... stay... strong! Natural Language Semantics, 18(4), 351383 .

91. Walton, D. (1990). What is reasoning? what is an argument? The Journal of Philosophy, 87(8), 399419.

92. Webber, B. L. (2016). A formal approach to discourse anaphora. Routledge.

93. Willer, M. (2013). Dynamics of epistemic modality. Philosophical Review, 122(1), 45-92.

94. Willer, M. (2015). An update on epistemic modals. Journal of Philosophical Logic, 44(6), 835-849.

95. Yalcin, S. (2012). Context probabilism. In Logic, language and meaning (pp. 12-21). Springer.

Publisher's Note Springer Nature remains neutral with regard to jurisdictional claims in published maps and institutional affiliations. 